\title{
INVARIANT MANIFOLDS FOR PARABOLIC EQUATIONS UNDER PERTURBATION OF THE DOMAIN
}

\author{
PARINYA SA NGIAMSUNTHORN \\ School of Mathematics and Statistics \\ University of Sydney, NSW 2006 Australia
}

\begin{abstract}
We study the effect of domain perturbation on invariant manifolds for semilinear parabolic equations subject to Dirichlet boundary condition. Under Mosco convergence assumption on the domains, we prove the upper and lower semicontinuity of both the local unstable invariant manifold and the local stable invariant manifold near a hyperbolic equilibrium. The continuity results are obtained by keeping track of the construction of invariant manifolds in P. W. Bates and C. K. R. T. Jones [Dynam. Report. Ser. Dynam. Systems Appl. Vol. 2, 1-38, 1989].
\end{abstract}

\section{INTRODUCTION}

The study of invariant manifolds is an important tool to understand the behaviour of a dynamical system near an equilibrium point. In this paper, we are interested in dynamical systems arising from semilinear parabolic equations. Let $\Omega$ be a bounded open set in $\mathbb{R}^{N}, N \geq 2$. We consider the parabolic equation of the form

$$
\left\{\begin{aligned}
\frac{\partial u}{\partial t}+\mathcal{A} u & =g(x, u) & & \text { in } \Omega \times(0, \infty) \\
u & =0 & & \text { on } \partial \Omega \times(0, \infty) \\
u(\cdot, 0) & =u_{0} & & \text { in } \Omega,
\end{aligned}\right.
$$

where $g$ is a function in $C\left(\mathbb{R}^{N} \times \mathbb{R}\right)$ and $\mathcal{A}$ is an elliptic operator. Our aim is to study how dynamics of the parabolic equation (1) changes when we vary the domain $\Omega$. In particular, we wish to establish the continuity of invariant manifolds with respect to the domain. We will consider a sequence of uniformly bounded domains

E-mail address: pasa4391@uni.sydney.edu.au .

Date: August 29, 2011.

2010 Mathematics Subject Classification. Primary 37L05; Secondary 35K58, 35B20.

Key words and phrases. domain perturbation, invariant manifolds, upper and lower semicontinuity, semilinear parabolic equations, Mosco convergence. 
$\Omega_{n}$ in $\mathbb{R}^{N}$ as a perturbation of $\Omega$. The perturbation of (1) is given by

$$
\left\{\begin{aligned}
\frac{\partial u}{\partial t}+\mathcal{A}_{n} u & =g_{n}(x, u) & & \text { in } \Omega_{n} \times(0, \infty) \\
u & =0 & & \text { on } \partial \Omega_{n} \times(0, \infty) \\
u(\cdot, 0) & =u_{0, n} & & \text { in } \Omega_{n} .
\end{aligned}\right.
$$

We impose conditions on the nonlinearities $g_{n}$ and $g$ so that the corresponding abstract parabolic equations

$$
\left\{\begin{aligned}
\dot{u}(t)+A_{n} u(t) & =f_{n}(u(t)) \quad t \in(0, \infty) \\
u(0) & =u_{0, n}
\end{aligned}\right.
$$

where $f_{n}(u)(x):=g_{n}(x, u(x))$ and

$$
\left\{\begin{aligned}
\dot{u}(t)+A u(t) & =f(u(t)) \quad t \in(0, \infty) \\
u(0) & =u_{0},
\end{aligned}\right.
$$

where $f(u)(x):=g(x, u(x))$ are well-posed in $L^{2}\left(\Omega_{n}\right)$ and $L^{2}(\Omega)$, respectively. In addition, we assume that $f_{n}(u)$ and $f(u)$ are higher order terms, that is, we will consider (3) and (4) as the linearised systems near an equilibrium (see Assumption 2.3).

In this work, we focus on singular perturbations of the domain, e.g. its topology changes, so that it is not possible in general to apply a change of variables (coordinate transform) to change the perturbed equation into an equivalent problem over the same spatial domain $\Omega$. This means that our class of domain perturbations cannot be reduced to a classical perturbation for the coefficients. Common examples include a sequence of dumbbell shape domains with shrinking handle and a sequence of domains with cracks. One of the main difficulties to establish the persistence result under domain perturbation is that the solutions of parabolic equations belong to different spaces, namely, $L^{2}\left(\Omega_{n}\right)$ and consequently the dynamical systems (semiflows) induced by these parabolic equations act on different spaces.

It is well-known from the theory of dynamical systems that hyperbolicity of an equilibrium is the main concept for persistence under small perturbations. We show in this paper that this principle is also valid for singular domain perturbation. Our main result states that under a suitable rather general class of domain perturbation, if the unperturbed system (4) has a local stable and a local unstable invariant manifolds in a neighbourhood of an equilibrium and the equilibrium is hyperbolic, then the perturbed system (3) also has a local stable and a local unstable invariant manifolds for $n$ sufficiently large. Moreover, we have the continuity (upper and lower semicontinuity) of these invariant manifolds with respect to the domain (see Theorem 2.5 and Theorem 2.6). This result is new.

There are similar results on the effect of domain variation on the dynamics of parabolic equations. In [15], upper semicontinuity of attractors is obtained for reaction-diffusion equations with Neumann boundary condition when the domain 
$\Omega \subset \mathbb{R}^{M} \times \mathbb{R}^{N}$ is squeezed in the $\mathbb{R}^{N}$-direction. Arrieta and Carvalho [3] consider a similar problem on a sequence of bounded and Lipschitz perturbed domains $\Omega_{n}$. They give necessary and sufficient conditions on domains for spectral convergence of the corresponding elliptic problem and obtain continuity (upper and lower semicontinuity) of local unstable manifolds and consequently continuity of attractors. For results under Dirichlet boundary condition, we refer to [9] where upper and lower semicontinuity of attractors are obtained for the heat equation under a certain perturbation of the domain in $\mathbb{R}^{N}$ with $N \leq 4$.

The class of domain perturbations considered in this paper (Assumption 2.2) is much more general than that in [9]. Many examples where this more general domain convergence is useful appear in [6] (as well as many other references). These have been used in constructing many examples of domains where the time independent problem is much more complicated than when $\Omega$ is a ball. For this general class of domain perturbations, we also have prior knowledge of the convergence of eigenvalues and eigenfunctions of the corresponding elliptic operators. The main focus here is to investigate the dependence of domains in the construction of invariant manifolds. In [3], continuity of local unstable invariant manifolds is proved by keeping track of the construction adapted from Henry [11]. Although our framework on semilinear parabolic equations fits into [11], we will use different techniques. Indeed, we apply the existence results for invariant manifolds in Bates and Jones [4] to prove the continuity of invariant manifolds under domain perturbation. The construction of invariant manifolds in [4] follows Hadamard style [10] which involves using the splitting between various subspaces to estimate projections of the flow in the different directions. The technique involves more geometrical than functional-analytic arguments. By using this construction, we give continuity results for both the local stable and the local unstable invariant manifolds under domain perturbation rather than focus only on the local unstable invariant manifolds (and consequently attractors) as in $[3,9,15]$.

An outline of this paper is as follows. In Section 2, we state our framework and the main results on the continuity (upper and lower semicontinuity) of the local stable and the local unstable invariant manifolds under perturbation of the domain. In Section 3, we obtain the existence of local invariant manifolds for the perturbed problems following the construction from [4]. In Section 4, we give some technical lemmas and a characterisation of upper and lower semicontinuity. The proof of the continuity results is given in Section 5 for the local unstable invariant manifolds and in Section 6 for the local stable invariant manifolds.

\section{Framework And MAin RESUlts}

Let $\Omega_{n}$ be a sequence of bounded open sets in $\mathbb{R}^{N}, N \geq 2$ and $\Omega$ be a bounded open set in $\mathbb{R}^{N}$ such that there exists a ball $D \subset \mathbb{R}^{N}$ with $\Omega_{n}, \Omega \subset D$ for all $n \in \mathbb{N}$. We consider the perturbed semilinear parabolic equation (2) where $\mathcal{A}_{n}$ is an elliptic 
operator of the form

$$
\mathcal{A}_{n} u:=-\partial_{i}\left[a_{i j, n}(x) \partial_{j} u+a_{i, n}(x) u\right]+b_{i, n}(x) \partial_{i} u+c_{0, n}(x) u .
$$

In the above, we use summation convention with $i, j$ running from 1 to $N$. Also, we assume $a_{i j, n}, a_{i, n}, b_{i, n}, c_{0, n}$ are functions in $L^{\infty}(D)$ and that there exists a constant $\alpha_{0}>0$ independent of $x \in D$ and $n \in \mathbb{N}$ such that

$$
a_{i j, n}(x) \xi_{i} \xi_{j} \geq \alpha_{0}|\xi|^{2},
$$

for all $\xi \in \mathbb{R}^{N}$ and for all $n \in \mathbb{N}$. The elliptic operator $\mathcal{A}$ for the unperturbed equation (1) is defined similarly to (5) (with $n$ deleted) and $a_{i j}$ satisfies the ellipticity condition (6) with the same constant $\alpha_{0}$. We assume that the coefficients of the operator $\mathcal{A}_{n}$ converge to the corresponding coefficients of $\mathcal{A}$ as stated below.

Assumption 2.1. Assume that $\lim _{n \rightarrow \infty} a_{i j, n}=a_{i j}, \lim _{n \rightarrow \infty} a_{i, n}=a_{i}, \lim _{n \rightarrow \infty} b_{i, n}=$ $b_{i}$ and $\lim _{n \rightarrow \infty} c_{0, n}=c_{0}$ in $L^{\infty}(D)$ for all $i, j=1, \ldots, N$.

By Riesz representation theorem, we identify $L^{2}\left(\Omega_{n}\right)$ with its dual and consider the evolution triple $H_{0}^{1}\left(\Omega_{n}\right) \stackrel{d}{\hookrightarrow} L^{2}\left(\Omega_{n}\right) \stackrel{d}{\hookrightarrow} H^{-1}\left(\Omega_{n}\right)$. We denote by $\langle\cdot, \cdot\rangle$ the duality pair between $H^{-1}\left(\Omega_{n}\right)$ and $H_{0}^{1}\left(\Omega_{n}\right)$. The notation $(\cdot \mid \cdot)_{L^{2}\left(\Omega_{n}\right)}$ denotes the inner product on $L^{2}\left(\Omega_{n}\right)$. Define a form $a_{n}(\cdot, \cdot)$ associated with $\mathcal{A}_{n}$ on $H_{0}^{1}\left(\Omega_{n}\right)$ by

$$
a_{n}(u, v):=\int_{\Omega_{n}}\left[a_{i j, n}(x) \partial_{j} u+a_{i, n}(x) u\right] \partial_{i} v+b_{i, n}(x) \partial_{i} u v+c_{0 . n}(x) u v d x,
$$

for $u, v \in H_{0}^{1}\left(\Omega_{n}\right)$. It is easy to see that $a_{n}(\cdot, \cdot)$ is a continuous bilinear form. We define $a(\cdot, \cdot)$ on $H_{0}^{1}(\Omega)$ similarly. Let

$$
\lambda_{\mathcal{A}}:=\sup _{n \in \mathbb{N}}\left\{\left\|c_{0, n}^{-}\right\|_{\infty}+\frac{1}{2 \alpha_{0}} \sum_{i=1}^{N}\left\|a_{i, n}+b_{i, n}\right\|_{\infty}\right\}
$$

where $c_{0, n}^{-}:=\max \left(-c_{0 . n}, 0\right)$ is the negative part of $c_{0, n}$. We set $\lambda_{0}:=\lambda_{\mathcal{A}}+\alpha_{0} / 2$. It can be verified that

$$
a_{n}(u, u)+\lambda\|u\|_{L^{2}\left(\Omega_{n}\right)}^{2} \geq \frac{\alpha_{0}}{2}\|u\|_{H_{0}^{1}\left(\Omega_{n}\right)}^{2},
$$

for all $u \in H_{0}^{1}\left(\Omega_{n}\right)$, for all $\lambda \geq \lambda_{0}$ and for all $n \in \mathbb{N}$. Similar inequality holds for $a(\cdot, \cdot)$ with the same constants. By the Lax-Milgram theorem, there exists $A_{\Omega_{n}} \in \mathscr{L}\left(H_{0}^{1}\left(\Omega_{n}\right), H^{-1}\left(\Omega_{n}\right)\right)$ such that

$$
a_{n}(u, v)=\left\langle A_{\Omega_{n}} u, v\right\rangle,
$$

for all $u, v \in H_{0}^{1}\left(\Omega_{n}\right)$. We may consider $A_{\Omega_{n}}$ as an operator on $H^{-1}\left(\Omega_{n}\right)$ with the domain $H_{0}^{1}\left(\Omega_{n}\right)$. Similarly, we obtain the operator $A_{\Omega} \in \mathscr{L}\left(H_{0}^{1}(\Omega), H^{-1}(\Omega)\right)$. Let $A_{n}$ and $A$ be the maximal restriction of the operators $A_{\Omega_{n}}$ and $A_{\Omega}$ on $L^{2}\left(\Omega_{n}\right)$ and $L^{2}(\Omega)$, respectively. It is well-known that $-A_{n}$ generates a strongly continuous analytic semigroup $S_{n}(t), t \geq 0$ on $L^{2}\left(\Omega_{n}\right)$ (see [8, Proposition 3, XVII §6]). Similarly, we denote by $S(t), t \geq 0$ the semigroup on $L^{2}\left(\Omega_{n}\right)$ generated by $-A$. We shall consider the perturbation (2) of (1) in the abstract form (3) and (4) in $L^{2}\left(\Omega_{n}\right)$ and $L^{2}(\Omega)$, respectively. 
To deal with domain perturbation where the solutions belong to different function spaces, we usually consider the trivial extension, that is, the extension by zero on $D \backslash \Omega$. In abuse of notation, we often write $u \in L^{2}(D)$ for the trivial extension of a function $u \in L^{2}(\Omega)$. On the other hand, we write $u \in L^{2}(\Omega)$ for a function $u \in L^{2}(D)$ to represent its restriction to $\Omega$. In particular, when we write $u_{n} \rightarrow u$ in $L^{2}(D)$ for $u_{n} \in L^{2}\left(\Omega_{n}\right)$ we mean the trivial extensions converge in $L^{2}(D)$. The notation $\left.u_{n}\right|_{\Omega}$ where $u_{n} \in L^{2}\left(\Omega_{n}\right)$ means that $u_{n}$ is first extended by zero on $D \backslash \Omega_{n}$ and then restricted to $\Omega$. A similar interpretation applies to the notation $\left.u\right|_{\Omega_{n}}$ when $u \in L^{2}(\Omega)$. We will use this convention throughout the paper without further comment.

We assume that a sequence of domains $\Omega_{n}$ converges to $\Omega$ in the following sense.

Assumption 2.2. We assume the following two conditions hold:

(M1) For every $\phi \in H_{0}^{1}(\Omega)$, there exists $\phi_{n}$ in $H_{0}^{1}\left(\Omega_{n}\right)$ such that $\phi_{n} \rightarrow \phi$ in $H^{1}(D)$.

(M2) If $\left(n_{k}\right)$ is a sequence of indices converging to $\infty,\left(\phi_{n_{k}}\right)$ is a sequence with $\phi_{n_{k}} \in H_{0}^{1}\left(\Omega_{n_{k}}\right)$ and $\phi_{n_{k}} \rightarrow \phi$ in $H^{1}(D)$ weakly, then the weak limit $u$ belongs to $H_{0}^{1}(\Omega)$.

Note that here we regard $H_{0}^{1}\left(\Omega_{n}\right)$ and $H_{0}^{1}(\Omega)$ as closed subspaces of $H^{1}(D)$ using the trivial extension. It is often said that $H_{0}^{1}\left(\Omega_{n}\right)$ converges to $H_{0}^{1}(\Omega)$ in the sense of Mosco when (M1) and (M2) hold, but we will simply say that $\Omega_{n}$ converges to $\Omega$ in sense of Mosco. We refer to [13] for a general Mosco convergence of closed convex sets. Examples of domains satisfying (M1) and (M2) can be found in [6].

The Mosco convergence assumption is naturally used in domain perturbation. As characterised in [6], it is a necessary and sufficient condition for strong convergence and uniform convergence of the resolvent operators under domain perturbation. It is also sufficient for the convergence of solutions of initial value problems for parabolic equations (see [7, Section 6]).

We make the following assumption on the nonlinearities.

Assumption 2.3. We assume that

(i) $f: L^{2}(\Omega) \rightarrow L^{2}(\Omega)$ is locally Lipschitz and $f(0)=0$. Moreover, for every $\varepsilon>0$ there exists a neighbourhood $U=U(\varepsilon)$ of 0 such that $f$ has a Lipschitz constant $\varepsilon$ in $U$.

(ii) $f_{n}: L^{2}\left(\Omega_{n}\right) \rightarrow L^{2}\left(\Omega_{n}\right)$ is locally Lipschitz and $f_{n}(0)=0$. In addition, for every $\varepsilon>0$ there exists a neighbourhood $U_{n}=U_{n}(\varepsilon)$ of 0 such that $f_{n}$ has a Lipschitz constant $\varepsilon$ in $U_{n}$. Moreover, $U_{n}$ can be chosen uniformly with respect to $n \in \mathbb{N}$ in the sense that we can take $U_{n}$ to be a ball centered at 0 in $L^{2}\left(\Omega_{n}\right)$ of the same radius for all $n \in \mathbb{N}$.

(iii) $f_{n}\left(\left.u\right|_{\Omega_{n}}\right) \rightarrow f\left(\left.u\right|_{\Omega}\right)$ in $L^{2}(D)$ uniformly with respect to $u \in B_{L^{2}(D)}(0, r)$ for all $r>0$.

Remark 2.4. (i) Assumption 2.3 (i) means that $f(u)$ is a higher order term and we could think of (4) as a linearised problem near an equilibrium. 
(ii) A necessary and sufficient condition for the substitution operator $f$ to be in $C\left(L^{p}\left(\mathbb{R}^{N}\right), L^{q}\left(\mathbb{R}^{N}\right)\right)$ is that there exist $c>0$ and $\psi \in L^{q}\left(\mathbb{R}^{N}\right)$ such that $|g(x, \xi)| \leq$ $\psi(x)+c|\xi|^{p / q}$ for all $x \in \mathbb{R}^{N}$ and $\xi \in \mathbb{R}$ (see [1]). Hence, Assumption 2.3 (i.e. $p=q=2$ ) means that we require a linear growth with respect to $u$ in the nonlinear term $g(x, u)$.

(iii) The Lipschitz continuity of $f$ is for instance satisfied if there exists an essentially bounded function $\phi$ such that $\left|g\left(x, \xi_{1}\right)-g\left(x, \xi_{2}\right)\right| \leq \phi(x, R)\left|\xi_{1}-\xi_{2}\right|$ for all $\left|\xi_{1}\right|,\left|\xi_{2}\right| \leq R$ (see [1, Theorem 3.10]).

(iv) The condition $f(0)=0$ holds if $g(x, 0)=0$ for almost all $x \in \Omega$.

By our assumptions on $A_{n}$ and $f_{n}$, the abstract equation (3) has a unique mild solution $u_{n} \in C\left(\left[0, t_{n}^{+}\left(u_{0, n}\right)\right), L^{2}\left(\Omega_{n}\right)\right)$ for any given initial condition $u_{0, n} \in L^{2}\left(\Omega_{n}\right)$ (see [14] or [7, Theorem 3.8]). Here, we write $t_{n}^{+}\left(u_{0, n}\right)$ for the maximal existence time or positive escape time. Moreover, the mild solution $u_{n}$ of (3) can be represented by the variation of constants formula

$$
u_{n}(t)=S_{n}(t) u_{0, n}+\int_{0}^{t} S_{n}(t-\tau) f_{n}\left(u_{n}(\tau)\right) d \tau,
$$

for $t \in\left[0, t_{n}^{+}\left(u_{0, n}\right)\right)$. Since $g_{n}$ is linearly bounded with respect to the second variable (Remark 2.4 (ii)), we have that $t_{n}^{+}\left(u_{0, n}\right)=\infty$ for all $u_{0, n} \in L^{2}\left(\Omega_{n}\right)$, that is, we always have a global solution. Similar consideration implies the existence and uniqueness of mild solution $u$ of (4).

To study the abstract parabolic equation as a dynamical system, we consider a semiflow $\Phi_{t, n}: L^{2}\left(\Omega_{n}\right) \rightarrow L^{2}\left(\Omega_{n}\right)$ defined by

$$
\Phi_{t, n}\left(u_{0, n}\right):=u_{n}(t)
$$

for all $t \in\left[0, t_{n}^{+}\left(u_{0, n}\right)\right)$ where $u_{n}$ is the maximal solution of (3). Sometimes we would like to study the backwards behaviour of solutions. We call a continuous curve $u_{n}:[-t, 0] \rightarrow L^{2}\left(\Omega_{n}\right)$ for some $t>0$ a backwards solution branch for $u_{0, n} \in$ $L^{2}\left(\Omega_{n}\right)$ if $\Phi_{s, n}\left(u_{n}(-s)\right)=u_{0, n}$ for all $s \in[0, t]$. We write $\Phi_{-s, n}\left(u_{0, n}\right)=u_{n}(-s)$ when we look at a particular backwards solution branch. We defined the semiflow $\Phi_{t}: L^{2}(\Omega) \rightarrow L^{2}(\Omega)$ induced by solutions of (4) similarly.

Under the assumptions considered above, it is proved in [4, Theorems 1.1 (i), 1.2 (i) ] that the unperturbed problem (4) has a local stable invariant manifold $W^{s}$ and a local unstable invariant manifold $W^{u}$ inside a suitable neighbourhood $U$ of 0 (see Section 3.1). In this paper, we study the persistence of these local invariant manifolds under domain perturbation when the equilibrium $0 \in L^{2}(\Omega)$ of (4) is hyperbolic, that is, the spectrum $\sigma(-A)$ of $-A$ does not contain $\lambda$ with $\operatorname{Re} \lambda=0$.

The main results of this paper can be stated as follows.

Theorem 2.5 (Continuity of local unstable manifolds). Suppose that Assumption 2.1, 2.2 and 2.3 are satisfied. If the equilibrium 0 of (4) is hyperbolic, then (3) has a local unstable invariant manifold $W_{n}^{u}$ for $n$ sufficiently large such that there exists $\delta>0$ for which the following (i) and (ii) hold. 
(i) Upper semicontinuity:

$$
\sup _{v \in W_{n}^{u} \cap B_{n}} \inf _{u \in W^{u} \cap B}\|v-u\|_{L^{2}(D)} \rightarrow 0 \quad \text { as } n \rightarrow \infty
$$

(ii) Lower semicontinuity:

$$
\sup _{u \in W^{u} \cap B} \inf _{v \in W_{n}^{u} \cap B_{n}}\|v-u\|_{L^{2}(D)} \rightarrow 0 \quad \text { as } n \rightarrow \infty,
$$

where $B_{n}:=B_{L^{2}\left(\Omega_{n}\right)}(0, \delta)$ and $B:=B_{L^{2}(\Omega)}(0, \delta)$.

A similar result can be stated for local stable invariant manifolds with an additional assumption of the convergence in measure of the domains. We denote by $|\Omega|$ the Lebesgue measure of $\Omega$.

Theorem 2.6 (Continuity of local stable manifolds). Suppose that Assumption 2.1, 2.2 and 2.3 are satisfied. In addition, assume that $\left|\Omega_{n}\right| \rightarrow|\Omega|$ as $n \rightarrow \infty$. If the equilibrium 0 of (4) is hyperbolic, then (3) has a local stable invariant manifold $W_{n}^{s}$ for $n$ sufficiently large such that there exists $\delta>0$ for which the following (i) and (ii) hold.

(i) Upper semicontinuity:

$$
\sup _{v \in W_{n}^{s} \cap B_{n}} \inf _{u \in W^{s} \cap B}\|v-u\|_{L^{2}(D)} \rightarrow 0 \quad \text { as } n \rightarrow \infty
$$

(ii) Lower semicontinuity:

$$
\sup _{u \in W^{s} \cap B} \inf _{v \in W_{n}^{s} \cap B_{n}}\|v-u\|_{L^{2}(D)} \rightarrow 0 \quad \text { as } n \rightarrow \infty,
$$

where $B_{n}:=B_{L^{2}\left(\Omega_{n}\right)}(0, \delta)$ and $B:=B_{L^{2}(\Omega)}(0, \delta)$.

\section{EXISTENCE OF INVARIANT MANIFOLDS FOR THE PERTURBED EQUATIONS}

In this section, we obtain the existence of local unstable and local stable invariant manifolds for the perturbed equation (3) stated in Theorem 2.5 and Theorem 2.6 using the construction from [4]. For the sake of mathematical necessity, we first give a sketch of proof of the existence of invariant manifolds proved in [4] for the unperturbed equation (4). We then keep track of this construction to obtain invariant manifolds for the perturbed equations.

\subsection{The construction of invariant manifolds.}

Definition 3.1. Let $U$ be a neighbourhood of 0 . We define

$W^{s}=\left\{u \in U: \Phi_{t}(u) \in U\right.$ for all $t \geq 0$ and $\Phi_{t}(u) \rightarrow 0$ exponentially as $\left.t \rightarrow \infty\right\}$

$W^{u}=\left\{u \in U\right.$ : some backwards branch $\Phi_{t}(u)$ exists for all $t<0$ and lies in $U$, and $\Phi_{t}(u) \rightarrow 0$ exponentially as $\left.t \rightarrow-\infty\right\}$ 
These sets $W^{s}$ and $W^{u}$ are invariant relative to $U$ and are called stable and unstable sets, respectively. Under the assumptions in Section 2, it is proved in [4] that $W^{s}$ and $W^{u}$ are indeed invariant manifolds for the unperturbed problem (4). We sometimes write $W^{s}(U)$ and $W^{u}(U)$ to indicate their dependence on the neighbourhood $U$.

Recall from Section 2 that $-A$ is a generator of an analytic $C_{0}$-semigroup $S(t), t \geq 0$ on $L^{2}(\Omega)$. We decompose the spectrum $\sigma(-A)$ as

$$
\sigma(-A)=\sigma^{s} \cup \sigma^{c} \cup \sigma^{u}
$$

where

$$
\begin{aligned}
\sigma^{s} & =\{\lambda \in \sigma(-A): \operatorname{Re}(\lambda)<0\} \\
\sigma^{c} & =\{\lambda \in \sigma(-A): \operatorname{Re}(\lambda)=0\} \\
\sigma^{u} & =\{\lambda \in \sigma(-A): \operatorname{Re}(\lambda)>0\} .
\end{aligned}
$$

Since $\Omega$ is bounded, Rellich's theorem implies that the embedding $H_{0}^{1}(\Omega) \hookrightarrow L^{2}(\Omega)$ is compact. Hence, the resolvent $(\lambda+A)^{-1}: L^{2}(\Omega) \rightarrow L^{2}(\Omega)$ is also compact when it is defined. This implies that $\sigma(-A)$ consists of eigenvalues with finite multiplicities (see [12]). It is easily seen from [8, Theorem 3, XVII $\S 6]$ that $\sigma^{c}$ and $\sigma^{u}$ are finite sets. Let $\Gamma^{c}$ and $\Gamma^{u}$ be rectifiable closed curves separating $\sigma^{c}$ and $\sigma^{s}$ respectively from the remaining spectrum. There are invariant subspaces of $L^{2}(\Omega)$ associated to $\sigma^{s}, \sigma^{c}$ and $\sigma^{u}$ via the spectral projections (see [12])

$$
P^{c}=\frac{1}{2 \pi i} \int_{\Gamma^{c}}(\lambda+A)^{-1} d \lambda \quad \text { and } \quad P^{u}=\frac{1}{2 \pi i} \int_{\Gamma^{u}}(\lambda+A)^{-1} d \lambda .
$$

Indeed, we decompose $L^{2}(\Omega)=X^{s} \oplus X^{c} \oplus X^{u}$ where $X^{s}=\left(1-P^{c}-P^{u}\right) L^{2}(\Omega)$, $X^{c}=P^{c} L^{2}(\Omega)$ and $X^{u}=P^{u} L^{2}(\Omega)$. Note that $\operatorname{dim}\left(X^{c}\right)$ and $\operatorname{dim}\left(X^{u}\right)$ are finite. We set $X^{c s}=X^{c} \oplus X^{s}$ and $X^{c u}=X^{c} \oplus X^{u}$. For $*=s, c, u, c s, c u$, we have that $-A^{*}=-\left.A\right|_{X^{*}}$ is a generator of $S^{*}(t)=\left.S(t)\right|_{X^{*}}$. Since $S(t)$ is an analytic semigroup, there exist $M>0$ and $\sigma>0$ such that $\left\|S^{s}(t)\right\| \leq M e^{-\sigma t}$ for all $t>0$.

To obtain the existence of local stable and unstable invariant manifolds, we decompose $L^{2}(\Omega)=X^{-} \oplus X^{+}$with $\operatorname{dim} X^{+}<\infty$ in two different ways; either $X^{-}=X^{s}$ and $X^{+}=X^{c u}$, or $X^{-}=X^{c s}$ and $X^{+}=X^{u}$. We denote a natural projection (via spectral projection) onto $X^{+}$by $P^{+}$, a natural projection on $X^{-}$ by $P^{-}:=1-P^{+}$and write $-A^{ \pm}=-\left.A\right|_{X^{ \pm}}$. In both cases, we have that $-A^{-}$ generates a $C_{0}$-semigroup $S^{-}(t)$ on $X^{-}$satisfying

$$
\left\|S^{-}(t)\right\| \leq M_{1} e^{\alpha t}
$$

for all $t \geq 0$ where $M_{1}>0$ and $\alpha \in \mathbb{R}$. Similarly, $-A^{+}$generates a $C_{0}$-group $S^{+}(t)$ on $X^{+}$satisfying

$$
\left\|S^{+}(t)\right\| \leq M_{2} e^{\beta t}
$$

for all $t \leq 0$ where $M_{2}>0$ and $\beta>\alpha$. The parameters $\alpha$ and $\beta$ can be chosen as follows (see proof of Theorem 1.1 case (D) and proof of Theorem 1.2 case (D) in [4]). 
- If $X^{-}=X^{s}$ and $X^{+}=X^{c u}$, we take $\alpha=-\sigma$ and fix $\beta$ such that $-\sigma<$ $\beta<0$.

- If $X^{-}=X^{c s}$ and $X^{+}=X^{u}$, we take $\beta>0$ such that $\beta<\min \{\operatorname{Re}(\lambda): \lambda \in$ $\sigma^{u}$ \} and fix $\alpha$ such that $0<\alpha<\beta$.

The main techniques used in [4] are a renorming of $X^{-}$and $X^{+}$and a modification of nonlinearity $f$. Since we decompose $L^{2}(\Omega)=X^{-} \oplus X^{+}$, norms on $X^{-}$and $X^{+}$ are originally inherited from $L^{2}(\Omega)$. Indeed, if $u=v \oplus w \in L^{2}(\Omega)$ where $v \in X^{-}$ and $w \in X^{+}$, then

$$
\frac{1}{\left\|P^{-}\right\|+\left\|P^{+}\right\|}\left(\|v\|_{L^{2}(\Omega)}+\|w\|_{L^{2}(\Omega)}\right) \leq\|u\|_{L^{2}(\Omega)} \leq\left(\|v\|_{L^{2}(\Omega)}+\|w\|_{L^{2}(\Omega)}\right) .
$$

However, we can renorm $X^{-}$and $X^{+}$by

$$
\begin{aligned}
\|v\|_{X^{-}}:=\sup _{t \geq 0} e^{-\alpha t}\left\|S^{-}(t) v\right\|_{L^{2}(\Omega)} & \text { for } v \in X^{-}, \\
\|w\|_{X^{+}}:=\sup _{t \leq 0} e^{-\beta t}\left\|S^{+}(t) w\right\|_{L^{2}(\Omega)} & \text { for } w \in X^{+} .
\end{aligned}
$$

These norms are equivalent on $X^{-}$and $X^{+}$, respectively. It is easy to see that (see also [4, Lemma 2.1] )

$$
\begin{array}{ll}
\|v\|_{L^{2}(\Omega)} \leq\|v\|_{X^{-}} \leq M_{1}\|v\|_{L^{2}(\Omega)} & \text { for all } v \in X^{-}, \\
\|w\|_{L^{2}(\Omega)} \leq\|w\|_{X^{+}} \leq M_{2}\|w\|_{L^{2}(\Omega)} & \text { for all } w \in X^{+} .
\end{array}
$$

The modification of nonlinearity $f$ is done by cutting off arguments so that we obtain a globally Lipschitz function $\tilde{f}$. Let $\eta>0$ be arbitrary. By Assumption 2.3 , we can choose $\delta>0$ such that $f$ has a Lipschitz constant less than $\eta / 12$ in $B_{L^{2}(\Omega)}(0,2 \delta)$. Let $\Psi: L^{2}(\Omega) \rightarrow \mathbb{R}$ be a function defined by

$$
\Psi(u)= \begin{cases}1 & \text { if }\|u\|_{L^{2}(\Omega)} \leq \delta \\ 2-\frac{\|u\|_{L^{2}(\Omega)}}{\delta} & \text { if } \delta \leq\|u\|_{L^{2}(\Omega)} \leq 2 \delta \\ 0 & \text { if }\|u\|_{L^{2}(\Omega)} \geq 2 \delta .\end{cases}
$$

By setting $\tilde{f}(u):=\Psi(u) f(u)$ for all $u \in L^{2}(\Omega)$, we have that $\tilde{f}$ is globally Lipschitz continuous with constant $\varepsilon<\eta / 4$. This Lipschitz constant $\varepsilon$ can be chosen as small as we require by shrinking $\delta$.

With this modified system $\dot{u}(t)+A u(t)=\tilde{f}(u(t))$, the solution to an initial value parabolic equation $u(t)$ also exists for $t \geq 0$, that is, the maximal existence time $t^{+}\left(u_{0}\right)=\infty$ for all $u_{0} \in L^{2}(\Omega)$. Moreover, the modified system agrees with the original system (4) inside $B_{L^{2}(\Omega)}(0, \delta)$. Hence, the modification gives us a local behaviour of the original system.

In [4], invariant manifolds for the modified system are constructed as follows. We choose the Lipschitz constant $\varepsilon$ of $\tilde{f}$ so that $\varepsilon<(\beta-\alpha) / 4$ and there exists $\gamma$ such that

$$
-\beta+2 \varepsilon<\gamma<-\alpha-2 \varepsilon .
$$


By abuse of notations, we denote again by $\Phi_{t}\left(u_{0}\right)$ the solution $u(t)$ of the modified system with the initial condition $u_{0}$. Let

$$
\begin{aligned}
& W^{-}=\left\{u \in L^{2}(\Omega): e^{\gamma t} \Phi_{t}(u) \rightarrow 0 \text { as } t \rightarrow \infty\right\} \\
& W^{+}=\left\{u \in L^{2}(\Omega): \text { a backward branch } \Phi_{t}(u) \text { exists for all } t \leq 0\right. \\
&\left.\quad \text { and } e^{\gamma t} \Phi_{t}(u) \rightarrow 0 \text { as } t \rightarrow-\infty\right\} .
\end{aligned}
$$

The main idea to show that $W^{-}$and $W^{+}$are invariant manifolds is that certain cones and moving cones are positively invariant, which can be determined by the difference in the growth rates on $X^{-}$and $X^{+}$. For $\lambda>0$, we define a cone

$$
K_{\lambda}=\left\{(v, w) \in X^{-} \times X^{+}: \lambda\|v\|_{X^{-}} \leq\|w\|_{X^{+}}\right\} .
$$

It is shown in [4, Lemma 2.4] that $K_{\lambda}$ is positively invariant if $\lambda \in[\mu, \nu]$ where $\mu$ and $\nu$ are positive parameters with $\mu<1<\nu$ satisfying

$$
\varepsilon<(\beta-\alpha) /\left(2+\nu+\mu^{-1}\right) .
$$

Indeed, $\mu$ and $\nu$ can be further restricted so that

$$
\varepsilon\left(1+\mu^{-1}\right)-\beta<\gamma<-\varepsilon(1+\nu)-\alpha .
$$

The next two theorems give the existence of global stable and global unstable invariant manifolds for the modified system. For the sake of mathematical necessity (when investigating the dependence on the domains), we sketch the proofs here.

Theorem 3.2 ( [4, Theorem 2.1]). There exists a Lipschitz function $h^{-}: X^{-} \rightarrow$ $X^{+}$such that $W^{-}=\operatorname{graph}\left(h^{-}\right)$and $h^{-}(0)=0$.

Sketch of the proof. Fix $v_{0} \in X^{-}$and let

$$
B=\left\{w_{0} \in X^{+}:\left\|w_{0}\right\|_{X^{+}} \leq \mu\left\|v_{0}\right\|_{X^{-}}\right\} .
$$

We write $\Phi_{t}\left(u_{0}\right)=u(t)$ as $u(t)=v(t) \oplus w(t)$ where $v(t) \in X^{-}$and $w(t) \in X^{+}$. Define

$$
G_{t}=\left\{w_{0} \in B:\|w(t)\|_{X^{+}} \leq \mu\|v(t)\|_{X^{-}}\right\} .
$$

It can be shown that $G_{\infty}:=\bigcap_{t \geq 0} G_{t}$ contains exactly one element. A function $h^{-}$ defined by $h^{-}\left(v_{0}\right)=G_{\infty}$ for $v_{0} \in X^{-}$is a Lipschitz function with $h^{-}(0)=0$ and $\operatorname{graph}\left(h^{-}\right)=W^{-}$.

Theorem 3.3 ( $\left[4\right.$, Theorem 2.2]). There exists a Lipschitz function $h^{+}: X^{+} \rightarrow$ $X^{-}$such that $W^{+}=\operatorname{graph}\left(h^{+}\right)$and $h^{+}(0)=0$.

Sketch of the proof. The proof is based on a standard contraction mapping argument. Let

$$
Y=\left\{h \in C\left(X^{+}, X^{-}\right): h(0)=0 \text { and } h \text { is } \nu^{-1} \text {-Lipschitz }\right\} .
$$

Then $Y$ is a complete metric space with the norm

$$
\|h\|_{\text {Lip }}=\sup _{w \neq 0} \frac{\|h(w)\|_{X^{-}}}{\|w\|_{X^{+}}} .
$$


For an arbitrary $h \in Y$, it can be shown that $P^{+} \Phi_{t}(\operatorname{graph}(h))=X^{+}$and that $\Phi_{t}(\operatorname{graph}(h))$ is the graph of a $\nu^{-1}$-Lipschitz function for all $t \geq 0$. Hence, the map $T_{t}: Y \rightarrow Y$ for $t \geq 0$ given by

$$
T_{t}(h)=\tilde{h}
$$

where $\tilde{h} \in Y$ with $\operatorname{graph}(\tilde{h})=\Phi_{t}(\operatorname{graph}(h))$ is well-defined. Furthermore, $T_{t}$ is a contraction on $Y$ for $t$ sufficiently large. Indeed,

$$
\left\|T_{t}\left(h_{2}\right)-T_{t}\left(h_{1}\right)\right\|_{\text {Lip }} \leq \nu(\nu-\mu)^{-1} \exp \left(\left(\alpha-\beta+\varepsilon\left(2+\mu+\nu^{-1}\right)\right) t\right)\left\|h_{2}-h_{1}\right\|_{\text {Lip }} .
$$

Hence, there exists a unique fixed point $h_{t} \in Y$ for $t$ sufficiently large. We can show that $h_{t}$ is a fixed point of $T_{\tau}$ for all $\tau \geq 0$ and $h^{+}:=h_{t}$ is the required Lipschitz function with $\operatorname{graph}\left(h^{+}\right)=W^{+}$and $h^{+}(0)=0$.

Remark 3.4. Let $Y_{0}=\{h \in Y: h$ is differentiable at 0 and $D h(0)=0\}$. Then $Y_{0}$ is closed in $Y$. As $D \tilde{f}(0)=0$ (in fact $D f(0)=0$ from Assumption 2.3), it can be shown that $T_{t}: Y_{0} \rightarrow Y_{0}$ for all $t>0$. Hence, the fixed point $h^{+}$in Theorem 3.3 lies on $Y_{0}$ (see the proposition after the proof of Theorem 2.2 in [4]).

The next two theorems give the existence of the local stable and the local unstable invariant manifolds for (4).

Theorem 3.5 ( [4, Theorem 1.1(i)]). Under the assumptions given above, there exists an open neighbourhood $U$ of 0 in $L^{2}(\Omega)$ such that $W^{s}$ is a Lipschitz manifold which is tangent to $X^{s}$ at 0 , that is, there exists a Lipschitz function $h^{s}$ : $P^{s}(U) \rightarrow X^{c u}$ such that $\operatorname{graph}\left(h^{s}\right)=W^{s}, h^{s}(0)=0$ and $h^{s}$ is differentiable at 0 with $D h^{s}(0)=0$.

Sketch of the proof. Set $X^{-}=X^{s}$ and $X^{+}=X^{c u}$. We take $\alpha=-\sigma$ and fix $\beta$ such that $-\sigma<\beta<0$. Renorm $X^{-}$and $X^{+}$by (18). By Assumption 2.3, there exists $\delta>0$ such that the modification $\tilde{f}$ has a Lipschitz constant $\varepsilon<(\beta-\alpha) / 4$ and the modified system agrees with the original system on $B_{L^{2}(\Omega)}(0, \delta)$. By applying Theorem 3.2, we can find a product neighbourhood $U \subset B_{L^{2}(\Omega)}(0, \delta)$ and prove that $W^{s}=W^{-} \cap U$ is a local stable invariant manifold. It can be shown that any local stable manifold constructed using another renorming and modification agrees on a neighbourhood on which the manifolds are both defined. The tangency condition $D h^{s}(0)=0$ follows by making $\mu \rightarrow 0$ (by letting $\varepsilon \rightarrow 0$ and possibly shrinking $U)$.

Theorem 3.6 ( [4, Theorem 1.2(i)]). Under the assumptions given above, there exists an open neighbourhood $U$ of 0 in $L^{2}(\Omega)$ such that $W^{u}$ is a Lipschitz manifold which is tangent to $X^{u}$ at 0 , that is, there exists a Lipschitz function $h^{u}: P^{u}(U) \rightarrow$ $X^{\text {cs }}$ such that $\operatorname{graph}\left(h^{u}\right)=W^{u}, h^{u}(0)=0$ and $h^{u}$ is differentiable at 0 with $D h^{u}(0)=0$.

Sketch of the proof. Set $X^{-}=X^{c s}$ and $X^{+}=X^{u}$. We take $\beta>0$ such that $\beta<\min \left\{\operatorname{Re}(\lambda): \lambda \in \sigma^{u}\right\}$ and fix $\alpha$ such that $0<\alpha<\beta$. Renorm $X^{-}$and $X^{+}$and modify the nonlinearity $f$ as in the proof of Theorem 3.5. Applying 
Theorem 3.3, we can find a product neighbourhood $U \subset B_{L^{2}(\Omega)}(0, \delta)$ and prove that $W^{u}=W^{+} \cap U$ is a local unstable invariant manifold. It can be shown that any local unstable manifold constructed using another renorming and modification agrees on a neighbourhood on which the manifolds are both defined. The tangency condition $D h^{s}(0)=0$ follows from Remark 3.4.

The product neigbourhood $U$ in Theorem 3.5 and Theorem 3.6 can be chosen to be $U=V_{1} \times V_{2}$ where $V_{1} \subset X^{-}$is a ball of radius $\delta_{1}$ and $V_{2} \subset X^{+}$is a ball of radius $\delta_{2}$ such that $\delta_{1}<\delta_{2}$ for the local stable manifold and $\delta_{1}>\delta_{2}$ for the local unstable manifold. In fact, with these choices of product neighbourhoods, $W^{s}$ is positively invariant and $W^{u}$ is negatively invariant ( see property (P4) in [4]).

3.2. Existence of invariant manifolds for the perturbed equations. In this section, we apply the construction of invariant manifold in Section 3.1 to obtain invariant manifolds for the perturbed equations (3) under the assumptions stated in Theorem 2.5 and Theorem 2.6. We first collect some preliminary results on domain perturbation for solutions of parabolic equations and the corresponding elliptic equations.

Under Mosco convergence (Assumption 2.2) and the uniform boundedness of the domains, it is known that if $\lambda \in \rho(-A)$, then $\lambda \in \rho\left(-A_{n}\right)$ for $n$ sufficiently large and $\left(\lambda+A_{n}\right)^{-1} \rightarrow(\lambda+A)^{-1}$ in $\mathscr{L}\left(L^{2}(D)\right)$ (see [6, Corollary 4.7]). An important consequence is stated in the following lemma.

Lemma 3.7 ( [6, Corollary 4.2]). Suppose that Assumption 2.1 and 2.2 are satisfied. If $\Sigma \subset \sigma(-A)$ is a compact spectral set and $\Gamma$ is a rectifiable closed curve enclosing $\Sigma$ and separating it from the remaining of spectrum, then $\sigma\left(-A_{n}\right)$ is separated by $\Gamma$ into a compact spectral set $\Sigma_{n}$ and the rest of spectrum for $n$ sufficiently large. Moreover, for the corresponding spectral projections $P$ and $P_{n}$, we have that the images of $P$ and $P_{n}$ have the same dimension and $P_{n}$ converges to $P$ in norm

We next consider the behaviour of solutions of the initial value problem (4) under domain perturbation. Recall from Remark 2.4 (ii) that Assumption 2.3 means $f$ is linear bounded with respect to $u$ and consequently the solution of (4) exists globally for any initial condition $u_{0} \in L^{2}(\Omega)$. We can state the convergence of solutions of parabolic equations under domain perturbation in terms of semiflows as follows.

Theorem 3.8. Suppose that Assumption 2.1, 2.2 and 2.3 are satisfied. Let $u_{0, n} \in$ $L^{2}\left(\Omega_{n}\right)$ and $u_{0} \in L^{2}(\Omega)$. If $\left.u_{0, n}\right|_{\Omega} \rightarrow u_{0}$ weakly in $L^{2}(\Omega)$, then

$$
\Phi_{t, n}\left(u_{0, n}\right) \rightarrow \Phi_{t}\left(u_{0}\right)
$$

in $L^{2}(D)$ as $n \rightarrow \infty$ uniformly with respect to $t \in\left(0, t_{0}\right]$ for all $t_{0} \in(0, \infty)$. Moreover, if $u_{0, n} \rightarrow u_{0}$ strongly in $L^{2}(D)$, then (25) holds uniformly with respect to $t \in\left[0, t_{0}\right]$ for all $t_{0} \in(0, \infty)$.

Proof. The assertion follows from similar arguments for the proof of [7, Theorem 6.5] (the case of $-\Delta$ ), that is, by applying [7, Theorem 4.6]. The only minor 
modification is that we need to rescale the elliptic forms $a_{n}(\cdot, \cdot)$ and $a(\cdot, \cdot)$ into coercive forms in order to apply [2, Theorem 5.2] to obtain the convergence of (degenerate) semigroups from the strong convergence of the resolvents. Note also that the convergence result under stronger assumptions on domains can be found in [5].

To construct invariant manifolds for the perturbed problem (3), we decompose $\sigma\left(-A_{n}\right)=\sigma_{n}^{s} \cup \sigma_{n}^{c} \cup \sigma_{n}^{u}$ where $\sigma_{n}^{s}, \sigma_{n}^{c}$ and $\sigma_{n}^{u}$ are sets defined similarly to (13). By Lemma 3.7, we have that $\Gamma^{c}$ and $\Gamma^{u}$ separate $\sigma_{n}^{c}$ and $\sigma_{n}^{u}$ respectively from the remaining of spectrum for $n$ sufficiently large. The hyperbolicity assumption $\left(\sigma^{c}=\emptyset\right)$ implies that $\sigma_{n}^{c}=\emptyset$ and hence the equilibrium $0 \in L^{2}\left(\Omega_{n}\right)$ of (3) is hyperbolic for all $n$ sufficiently large. We define the spectral projections $P_{n}^{c}$ and $P_{n}^{u}$ similarly to (14) and write $P_{n}^{s}:=1-P_{n}^{c}-P_{n}^{u}$. Note that the hyperbolicity assumption implies $P_{n}^{c}=0$ for $n$ sufficiently large. In addition, Lemma 3.7 implies that

$$
P_{n}^{c} \rightarrow P^{c} \quad \text { and } \quad P_{n}^{u} \rightarrow P^{u}
$$

in $\mathscr{L}\left(L^{2}(D)\right)$ as $n \rightarrow \infty$. We decompose

$$
L^{2}\left(\Omega_{n}\right)=X_{n}^{s} \oplus X_{n}^{c} \oplus X_{n}^{u},
$$

where $X_{n}^{s}, X_{n}^{c}$ and $X_{n}^{u}$ are the images of $P_{n}^{s}, P_{n}^{c}$ and $P_{n}^{u}$, respectively. From the above consideration we have that $X_{n}^{c}=\{0\}$ and $X_{n}^{u}$ is a finite dimensional subspace with $\operatorname{dim}\left(X_{n}^{u}\right)=\operatorname{dim}\left(X^{u}\right)$ for all $n$ sufficiently large.

In order to obtain invariant manifolds for the modified system of the perturbed equation (3), we decompose $L^{2}\left(\Omega_{n}\right)$ as $X_{n}^{-} \oplus X_{n}^{+}$in two different ways as in Section 3.1. In particular, $\operatorname{dim}\left(X_{n}^{+}\right)=\operatorname{dim}\left(X^{+}\right)<\infty$ for $n$ sufficiently large and

$$
P_{n}^{+} \rightarrow P^{+}
$$

in $\mathscr{L}\left(L^{2}(D)\right)$. By Assumption 2.1, we can choose the parameters $\alpha$ and $\beta$ for the restriction of semigroup $S_{n}(t)$ to $X_{n}^{-}$and $X_{n}^{+}$uniformly with respect to $n \in \mathbb{N}$ so that $S_{n}^{-}(t)$ and $S_{n}^{+}(t)$ satisfy similar estimates as in (15) and (16), respectively. We can renorm $X_{n}^{-}$and $X_{n}^{+}$using similar norms involving $S_{n}^{-}(t)$ and $S_{n}^{+}(t)$ as defined in (18). In particular, similar estimates as in (19) hold for the norms $\|\cdot\|_{X_{n}^{-}}$and $\|\cdot\|_{X_{n}^{+}}$with uniform constants $M_{1}$ and $M_{2}$ for $n$ sufficiently large.

By Assumption 2.3 (ii), there exists $\delta>0$ independent of $n$ such that the modification $\tilde{f}_{n}$ of $f_{n}$ has a Lipschitz constant $\varepsilon<(\beta-\alpha) / 4$ and the modified system agrees with the original system on $B_{n}:=B_{L^{2}\left(\Omega_{n}\right)}(0, \delta)$ for all $n \in \mathbb{N}$. Therefore, we can construct the stable and unstable invariant manifold for the modified system by using uniform parameters $\gamma, \mu$ and $\nu$ for all $n$ large. By Theorem 3.5, there exists a product neighbourhood $U_{n} \subset B_{n}$ such that a local stable invariant manifold is $W_{n}^{s}\left(U_{n}\right)=\operatorname{graph}\left(h_{n}^{-}\right) \cap U_{n}$. Since the parameters $\alpha$ and $\beta$ are chosen uniformly for the renorming of $X_{n}^{-}$and $X_{n}^{+}$respectively, we can choose $U_{n} \subset B_{n}$ to be a product neighbourhood $V_{1, n} \times V_{2, n}$ where $V_{1, n} \subset X_{n}^{-}$is a ball of radius $\delta_{1}$ and $V_{2, n} \subset X_{n}^{+}$is a ball of radius $\delta_{2}$ with $\delta_{1}<\delta_{2}$ for all $n \in \mathbb{N}$. Without loss of 
generality we may choose $\delta$ smaller so that the modified system agrees with the original system on $\bar{B}_{n}$ for all $n \in \mathbb{N}$. Similarly, by Theorem 3.6, there exists a product neighbourhood $\tilde{U}_{n} \subset B_{n}$ such that a local unstable invariant manifold is $W_{n}^{u}\left(\tilde{U}_{n}\right)=\operatorname{graph}\left(h_{n}^{+}\right) \cap \tilde{U}_{n}$. Since the parameters $\alpha$ and $\beta$ are chosen uniformly for the renorming of $X_{n}^{-}$and $X_{n}^{+}$respectively, we can choose $\tilde{U}_{n} \subset B_{n}$ to be a product neighbourhood $\tilde{V}_{1, n} \times \tilde{V}_{2, n}$ where $\tilde{V}_{1, n} \subset X_{n}^{-}$is a ball of radius $\tilde{\delta}_{1}$ and $\tilde{V}_{2, n} \subset X_{n}^{+}$ is a ball of radius $\tilde{\delta}_{2}$ with $\tilde{\delta}_{1}>\tilde{\delta}_{2}$ for all $n \in \mathbb{N}$. Again we may choose $\delta$ smaller so that the modified system agrees with the original system on $\bar{B}_{n}$ for all $n \in \mathbb{N}$. Therefore, we have established the existence of local unstable manifolds and local stable manifolds for the perturbed problem (3).

We can assume that the choice of neighbourhoods considered above applies to the limit problem (4) (by possibly shrinking $\delta$ ). Therefore, to prove Theorem 2.5 and Theorem 2.6, it remains to verify the continuity under domain perturbation (upper and lower semicontinuity) of local stable and local unstable invariant manifolds inside some ball $B_{n}=B_{L^{2}\left(\Omega_{n}\right)}(0, \hat{\delta})$ contained in $U_{n}=V_{1, n} \times V_{2, n}$ or $\tilde{U}_{n}=\tilde{V}_{1, n} \times$ $\tilde{V}_{2, n}$.

Remark 3.9. By our assumptions and the application of [2, Theorem 5.2], we know that $S_{n}(t)$ converges to $S(t)$ in the strong operator topology uniformly with respect to $t$ on compact subsets of $(0, \infty)$. The main difficulty to prove upper and lower semicontinuity of invariant manifolds using the construction in [4] is that we need to deal with sequences of functions under a sequence of the special norms $\|\cdot\|_{X_{n}^{-}}$and $\|\cdot\|_{X_{n}^{+}}$defined in terms of the supremum of $e^{-\alpha t}\left\|S_{n}^{-}(t) v\right\|_{L^{2}\left(\Omega_{n}\right)}$ on a non-compact interval $[0, \infty)$ and the supremum of $e^{-\beta t}\left\|S_{n}^{+}(t) w\right\|_{L^{2}\left(\Omega_{n}\right)}$ on $(-\infty, 0]$, respectively (see (18)). In particular, we do not generally have the convergence of a sequence of functions in $X_{n}^{-}$or $X_{n}^{+}$with respect to a sequence of the norms mentioned above.

\section{Some technical RESUlts TOWARDS THE PROOF OF SEMICONTINUITy}

In this section, we give some technical results required to prove upper and lower semicontinuity in Theorem 2.5 and Theorem 2.6. In particular, we prove some convergence result for a bounded sequence $\left(w_{n}\right)_{n \in \mathbb{N}}$ with $w_{n} \in X_{n}^{+}$for each $n \in \mathbb{N}$. Moreover, we give a characterization of upper and lower semicontinuity.

\subsection{Convergence of sequences in finite dimensional subspaces.}

Lemma 4.1. Let $\left(\phi_{n}\right)_{n \in \mathbb{N}}$ be a sequence with $\phi_{n} \in L^{2}\left(\Omega_{n}\right)$ for each $n \in \mathbb{N}$ and $\phi \in L^{2}(\Omega)$. We decompose $\phi_{n}:=\phi_{n}^{s} \oplus \phi_{n}^{c} \oplus \phi_{n}^{u}$ corresponding to the decomposition (27). Similarly, we decompose $\phi:=\phi^{s} \oplus \phi^{c} \oplus \phi^{u}$. If $\phi_{n} \rightarrow \phi$ strongly in $L^{2}(D)$, then $\phi_{n}^{*} \rightarrow \phi^{*}$ strongly in $L^{2}(D)$ for $*=s, c, u$.

Proof. A direct application of (26) implies $\phi_{n}^{c} \rightarrow \phi^{c}$ and $\phi_{n}^{u} \rightarrow \phi^{u}$ in $L^{2}(D)$. Since $\phi_{n}^{s}=\left(1-P_{n}^{c}-P_{n}^{u}\right) \phi_{n}$ and $\phi^{s}=\left(1-P^{c}-P^{u}\right) \phi$, we also get $\phi_{n}^{s} \rightarrow \phi^{s}$ in $L^{2}(D)$.

Remark 4.2. The convergence $\phi_{n}^{s} \rightarrow \phi^{s}$ in Lemma 4.1 is different to convergence of the projections $\left(1-P_{n}^{c}-P_{n}^{u}\right) \rightarrow\left(1-P^{c}-P^{u}\right)$ in $\mathscr{L}\left(L^{2}(D)\right)$. For example, 
consider a square domain $\Omega$ in $\mathbb{R}^{2}$ perturbed by attaching "fingers" to one of the sides. If we increase the number of fingers so that the measure remains the same (by letting their width go to zero). Then $\left|\Omega_{n} \backslash \Omega\right|$ is a positive constant for all $n \in \mathbb{N}$. It is known that $H_{0}^{1}\left(\Omega_{n}\right)$ converges to $H_{0}^{1}(\Omega)$ in the sense of Mosco (see [6, Example 8.4]). Let $f \in L^{2}(D)$ be the constant function 1 . By (26), we have that $P_{n}^{c} f \rightarrow P^{c} f$ and $P_{n}^{u} f \rightarrow P^{u} f$ in $L^{2}(D)$. If $\left(1-P_{n}^{c}-P_{n}^{u}\right) f \rightarrow\left(1-P^{c}-P^{u}\right) f$ in $L^{2}(D)$, then $\left.\left.f\right|_{\Omega_{n}} \rightarrow f\right|_{\Omega}$ in $L^{2}(D)$. This cannot be true because $\left\|\left.f\right|_{\Omega_{n}}-\left.f\right|_{\Omega}\right\|_{L^{2}(D)}=\left|\Omega_{n} \backslash \Omega\right|>0$ for all $n \in \mathbb{N}$. Hence, $\left(1-P_{n}^{c}-P_{n}^{u}\right)$ does not converge to $\left(1-P^{c}-P^{u}\right)$ in $\mathscr{L}\left(L^{2}(D)\right)$. Note that if we impose the assumption that the Lebesgue measure of the domain converges, that is, $\left|\Omega_{n}\right| \rightarrow|\Omega|$ as $n \rightarrow \infty$, then we obtain the convergence $\left(1-P_{n}^{c}-P_{n}^{u}\right) \rightarrow\left(1-P^{c}-P^{u}\right)$ in $\mathscr{L}\left(L^{2}(D)\right)$.

In the next few results, we consider an arbitrary finite dimensional subspace of $L^{2}\left(\Omega_{n}\right)$.

Lemma 4.3. Let $m$ be a positive integer. Suppose $V_{n}$ is an $m$-dimensional subspace of $L^{2}\left(\Omega_{n}\right)$ with a basis $\left\{f_{1, n}, f_{2, n}, \ldots f_{m, n}\right\}$ for each $n \in \mathbb{N}$, and $V$ is an $m$-dimensional subspace of $L^{2}(\Omega)$ with a basis $\left\{f_{1}, f_{2}, \ldots f_{m}\right\}$. If $f_{j, n} \rightarrow f_{j}$ in $L^{2}(D)$ as $n \rightarrow \infty$ for all $j=1, \ldots, m$, then there exists $\hat{c}>0$ such that

$$
c_{n}:=\inf \left\{\left\|\sum_{j=1}^{m} \xi_{j} f_{j, n}\right\|_{L^{2}\left(\Omega_{n}\right)}: \xi=\left(\xi_{1}, \ldots, \xi_{m}\right) \in \mathbb{R}^{m},|\xi|=1\right\} \geq \hat{c},
$$

for all $n \in \mathbb{N}$.

Proof. Let $\xi=\left(\xi_{1}, \ldots, \xi_{m}\right) \in \mathbb{R}^{m}$ with $|\xi|=1$. By convergence of the bases, we get

$$
\begin{aligned}
\left\|\sum_{j=1}^{m} \xi_{j} f_{j, n}-\sum_{j=1}^{m} \xi_{j} f_{j}\right\|_{L^{2}(D)} & \leq \sum_{j=1}^{m}\left|\xi_{j}\right|\left\|f_{j, n}-f_{j}\right\|_{L^{2}(D)} \\
& \leq \sum_{j=1}^{m}\left\|f_{j, n}-f_{j}\right\|_{L^{2}(D)} \\
& \rightarrow 0
\end{aligned}
$$

as $n \rightarrow \infty$. Notice that the above convergence does not depend on $\xi$. This means $\left\|\sum_{j=1}^{m} \xi_{j} f_{j, n}\right\|_{L^{2}\left(\Omega_{n}\right)} \rightarrow\left\|\sum_{j=1}^{m} \xi_{j} f_{j}\right\|_{L^{2}(\Omega)}$ uniformly with respect to $\xi \in \mathbb{R}^{m}$ with $|\xi|=1$. Let

$$
c:=\inf \left\{\left\|\sum_{j=1}^{m} \xi_{j} f_{j}\right\|_{L^{2}(\Omega)}: \xi=\left(\xi_{1}, \ldots, \xi_{m}\right) \in \mathbb{R}^{m},|\xi|=1\right\} .
$$

In particular, choosing $\zeta>0$ such that $c-\zeta>0$, there exists $N_{0} \in \mathbb{N}$ (independent of $\xi \in \mathbb{R}^{m}$ with $|\xi|=1$ ) such that

$$
\left\|\sum_{j=1}^{m} \xi_{j} f_{j, n}\right\|_{L^{2}\left(\Omega_{n}\right)} \geq\left\|\sum_{j=1}^{m} \xi_{j} f_{j}\right\|_{L^{2}(\Omega)}-\zeta
$$

for all $n>N_{0}$ and for all $\xi \in \mathbb{R}^{m}$ with $|\xi|=1$. Since $\left\|\sum_{j=1}^{m} \xi_{j} f_{j}\right\|_{L^{2}(\Omega)} \geq c$, it follows that $\left\|\sum_{j=1}^{m} \xi_{j} f_{j, n}\right\|_{L^{2}\left(\Omega_{n}\right)} \geq c-\zeta$ for all $n>N_{0}$ and for all $\xi \in \mathbb{R}^{m}$ with 
$|\xi|=1$. Taking the infimum over $\xi \in \mathbb{R}^{m}$ with $|\xi|=1$, we obtain $c_{n} \geq c-\zeta>0$ for all $n \geq N_{0}$. Finally, taking $\hat{c}:=\min \left\{c_{1}, \ldots c_{N_{0}}, c-\zeta\right\}$, the lemma follows.

An immediate application of Lemma 4.3 is the following result.

Corollary 4.4. Assume that $V_{n}$ and $V$ are as in Lemma 4.3 and that the convergence of bases $f_{j, n} \rightarrow f_{j}$ in $L^{2}(D)$ as $n \rightarrow \infty$ holds for all $j=1, \ldots, m$. Let $u_{n}$ be a sequence such that $u_{n} \in V_{n}$ for each $n \in \mathbb{N}$. If $\left\|u_{n}\right\|_{L^{2}\left(\Omega_{n}\right)}$ is uniformly bounded, then there exists a subsequence $u_{n_{k}}$ such that $u_{n_{k}} \rightarrow u$ in $L^{2}(D)$ with a limit $u \in V$.

Proof. For each $n \in \mathbb{N}$, we write $u_{n}=\sum_{j=1}^{m} \xi_{j, n} f_{j, n}$. By a standard argument in the proof of equivalence of norms for finite dimensional spaces,

$$
\sum_{j=1}^{m}\left|\xi_{j, n}\right| \leq \frac{m}{c_{n}}\left\|u_{n}\right\|_{L^{2}\left(\Omega_{n}\right)},
$$

for all $n \in \mathbb{N}$, where $c_{n}$ is given in Lemma 4.3. It follows from the uniform boundedness of $\left\|u_{n}\right\|_{L^{2}\left(\Omega_{n}\right)}$ and Lemma 4.3 that $\sum_{j=1}^{m}\left|\xi_{j, n}\right|$ is uniform bounded. We can extract a subsequence $\xi_{j, n_{k}}$ such that $\xi_{j, n_{k}} \rightarrow \xi_{j}$ for all $j=1, \ldots, m$. Hence, $u_{n_{k}} \rightarrow u:=\sum_{j=1}^{m} \xi_{j} f_{j}$ in $L^{2}(D)$.

Recall that we have $\operatorname{dim}\left(X_{n}^{+}\right)=\operatorname{dim}\left(X^{+}\right)<\infty$ for sufficiently large $n$. We set $d:=\operatorname{dim}\left(X^{+}\right)$and fix a certain basis $\left\{f_{1}, f_{2}, \ldots, f_{d}\right\}$ of $X^{+}$. Define

$$
f_{j, n}:=\left.P_{n}^{+} f_{j}\right|_{\Omega_{n}},
$$

for $j=1, \ldots, d$. Then we obtain a basis of $X_{n}^{+}$as shown below.

Theorem 4.5. There exists $N_{0} \in \mathbb{N}$ such that $\left\{f_{1, n}, f_{2, n}, \ldots, f_{d, n}\right\}$ where $f_{j, n}$ defined by (29) is a basis of $X_{n}^{+}$for each $n>N_{0}$. Moreover, $f_{j, n} \rightarrow f_{j}$ in $L^{2}(D)$ as $n \rightarrow \infty$ holds for all $j=1, \ldots, d$.

Proof. The convergence $f_{j, n} \rightarrow f_{j}$ is clear from the definition of $f_{j, n}$ and (28). Since $X_{n}^{+}$is $d$-dimensional subspace for all $n$ sufficiently large, it suffices to show that there exists $N_{0} \in \mathbb{N}$ such that $f_{1, n}, f_{2, n}, \ldots, f_{d, n}$ are linearly independent for each $n>N_{0}$. We prove this by using mathematical induction on $m$ for $m=1, \ldots, d$ in the following statement: there exists $N_{m} \in \mathbb{N}$ such that $f_{1, n}, f_{2, n}, \ldots, f_{m, n}$ are linearly independent for each $n>N_{m}$.

The statement is trivial for $m=1$. For the induction step, suppose that the statement is true for $1, \ldots, m$ with $m<d$, but there is no $N_{m+1} \in \mathbb{N}$ such that $f_{1, n}, f_{2, n}, \ldots, f_{m+1, n}$ are linearly independent for each $n>N_{m+1}$. Thus, we can extract a subsequence $n_{k}$ (choosing $n_{k}>N_{m}$ for all $k \in \mathbb{N}$ ) such that $f_{1, n_{k}}, f_{2, n_{k}}, \ldots, f_{m+1, n_{k}}$ are linearly dependent for all $k \in \mathbb{N}$. By the linear independence of $f_{1, n_{k}}, f_{2, n_{k}}, \ldots, f_{m, n_{k}}$, we can write $f_{m+1, n_{k}}=\sum_{j=1}^{m} \xi_{j, n_{k}} f_{j, n_{k}}$ for all $k \in \mathbb{N}$. Since $f_{m+1, n_{k}} \rightarrow f_{m+1}$ in $L^{2}(D)$ as $k \rightarrow \infty$, it follows that $\left\|f_{m+1, n_{k}}\right\|_{L^{2}\left(\Omega_{n_{k}}\right)}$ is uniformly bounded. Corollary 4.4 implies that there exists a subsequence denoted again by $f_{m+1, n_{k}}$ such that $f_{m+1, n_{k}} \rightarrow f$ in $L^{2}(D)$ as $k \rightarrow \infty$, where the limit $f$ belongs to the $m$-dimensional subspace spanned by $f_{1}, f_{2}, \ldots, f_{m}$. By the uniqueness 
of a limit, we conclude that $f_{m+1}=f$. This is a contradiction to the assumption that $\left\{f_{1}, f_{2}, \ldots, f_{d}\right\}$ is a basis of $X^{+}$. Hence, the induction statement is true for $m+1$ and the theorem is proved.

As a consequence, we obtain the following convergence of a bounded sequence with each term belongs to a sequence of the spaces $X_{n}^{+}$.

Corollary 4.6. Let $\left(w_{n}\right)_{n \in \mathbb{N}}$ be a sequence with $w_{n} \in X_{n}^{+}$for each $n \in \mathbb{N}$. If $\left\|w_{n}\right\|_{L^{2}\left(\Omega_{n}\right)}$ (or $\left\|w_{n}\right\|_{X_{n}^{+}}$) is uniformly bounded, then there exists a subsequence $w_{n_{k}}$ such that $w_{n_{k}} \rightarrow w$ in $L^{2}(D)$ with the limit $w \in X^{+}$.

Proof. The result follows immediately from Corollary 4.4 and Theorem 4.5 and the equivalence of norms on $X_{n}^{+}$.

Remark 4.7. The above result implies that there exists a subsequence $w_{n_{k}}$ such that $\left\|w_{n_{k}}\right\|_{L^{2}\left(\Omega_{n_{k}}\right)} \rightarrow\|w\|_{L^{2}(\Omega)}$ but does not implies $\left\|w_{n_{k}}\right\|_{X_{n_{k}}^{+}} \rightarrow\|w\|_{X^{+}}$as degenerate semigroup only converges uniformly on compact subsets of $(0, \infty)$.

4.2. Characterisation of upper and lower semicontinuity. We give some equivalent statements for upper and lower semicontinuity mentioned in Theorem 2.5 and Theorem 2.6. We simplify the notations by considering bounded subsets $W_{n}, W$ of $L^{2}(D)$.

Lemma 4.8 (Characterisation of upper semicontinuity). The following statements are equivalent.

(i) $\sup _{v \in W_{n}} \inf _{u \in W}\|v-u\|_{L^{2}(D)} \rightarrow 0$ as $n \rightarrow \infty$.

(ii) For any sequence $\left\{v_{n}\right\}_{n \in \mathbb{N}}$ with $v_{n} \in W_{n}$, we have $\inf _{u \in W}\left\|v_{n}-u\right\|_{L^{2}(D)} \rightarrow 0$ as $n \rightarrow \infty$.

(iii) For any sequence $\left\{v_{n}\right\}_{n \in \mathbb{N}}$ with $v_{n} \in W_{n}$, if $\left\{v_{n_{k}}\right\}_{k \in \mathbb{N}}$ is a subsequence, then there exist a further subsequence (denoted again by $v_{n_{k}}$ ) and a sequence $\left\{u_{n_{k}}\right\}_{k \in \mathbb{N}}$ with $u_{n_{k}} \in W$ such that $\left\|v_{n_{k}}-u_{n_{k}}\right\|_{L^{2}(D)} \rightarrow 0$ as $k \rightarrow \infty$.

Proof. The statement (i) $\Rightarrow$ (ii) is clear. For (ii) $\Rightarrow$ (i), we prove by contrapositive. Suppose that (i) fails. Then

$$
\limsup _{n \rightarrow \infty}\left\{\sup _{v \in W_{n}} \inf _{u \in W}\|v-u\|_{L^{2}(D)}\right\}=: a>0 .
$$

We can find a subsequence $n_{k} \rightarrow \infty$ such that $\sup _{v \in W_{n_{k}}} \inf _{u \in W}\|v-u\|_{L^{2}(D)} \rightarrow a$ as $k \rightarrow \infty$. This implies that there exists $v_{n_{k}} \in W_{n_{k}}$ such that

$$
\inf _{u \in W}\left\|v_{n_{k}}-u\right\|_{L^{2}(D)}>a / 2,
$$

for all $k \in \mathbb{N}$. Hence, (ii) fails.

For the statement (ii) $\Leftrightarrow$ (iii), notice first that $\inf _{u \in W}\left\|v_{n}-u\right\|_{L^{2}(D)} \rightarrow 0$ as $n \rightarrow$ $\infty$ if and only if there exists $u_{n} \in W$ such that $\left\|v_{n}-u_{n}\right\|_{L^{2}(D)} \rightarrow 0$ as $n \rightarrow \infty$. To see this, we choose $u_{n} \in W$ such that $\left\|v_{n}-u_{n}\right\|_{L^{2}(D)}<\inf _{u \in W}\left\|v_{n}-u\right\|_{L^{2}(D)}+1 / n$ for each $n \in \mathbb{N}$. Then the forward implication follows. The backward implication is 


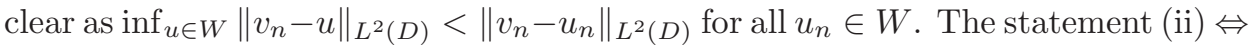
(iii) then simply follows from the above and a standard subsequence characterisation of a limit.

By a similar argument, we can state the following lemma.

Lemma 4.9 (Characterisation of lower semicontinuity). The following statements are equivalent.

(i) $\sup _{u \in W} \inf _{v \in W_{n}}\|v-u\|_{L^{2}(D)} \rightarrow 0$ as $n \rightarrow \infty$.

(ii) For any sequence $\left\{u_{n}\right\}_{n \in \mathbb{N}}$ with $u_{n} \in W$, we have inf $v_{v \in W_{n}}\left\|v-u_{n}\right\|_{L^{2}(D)} \rightarrow$ 0 as $n \rightarrow \infty$.

(iii) For any sequence $\left\{u_{n}\right\}_{n \in \mathbb{N}}$ with $u_{n} \in W$, if $\left\{u_{n_{k}}\right\}_{k \in \mathbb{N}}$ is a subsequence, then there exist a further subsequence (denoted again by $u_{n_{k}}$ ) and a sequence $\left\{v_{n_{k}}\right\}_{k \in \mathbb{N}}$ with $v_{n_{k}} \in W_{n_{k}}$ such that $\left\|v_{n_{k}}-u_{n_{k}}\right\|_{L^{2}(D)} \rightarrow 0$ as $k \rightarrow \infty$.

\section{Convergence of Unstable invariant MANifoldS}

In this section, we prove upper and lower semicontinuity of local unstable invariant manifolds. We first show pointwise convergence of global unstable manifolds for the modified systems in Section 5.1. Consequently, we prove Theorem 2.5 in Section 5.2.

\subsection{Convergence of global unstable manifolds. Let}

$$
Y_{n}=\left\{h \in C\left(X_{n}^{+}, X_{n}^{-}\right): h(0)=0 \text { and } h \text { is } \nu^{-1} \text {-Lipschitz }\right\} .
$$

Then $Y_{n}$ is a complete metric space with the norm

$$
\|h\|_{\text {Lip }}=\sup _{w \neq 0} \frac{\|h(w)\|_{X_{n}^{-}}}{\|w\|_{X_{n}^{+}}} .
$$

We define $T_{t, n}: Y_{n} \rightarrow Y_{n}$ for $t \geq 0$ by $T_{t, n}(h)=\tilde{h}$ where $\tilde{h} \in Y_{n}$ such that $\operatorname{graph}(\tilde{h})=\Phi_{t, n}(\operatorname{graph}(h))$. Fix $t>0$ sufficiently large such that

$$
K:=\nu(\nu-\mu)^{-1} \exp \left(\left(\alpha-\beta+\varepsilon\left(2+\mu+\nu^{-1}\right)\right) t\right)<1 .
$$

As in Theorem 3.3, $T_{t, n}$ is a contraction on $Y_{n}$ with a uniform contraction constant $K$ for all $n \in \mathbb{N}$. Moreover, $W_{n}^{+}$is a graph of the fixed point $h_{n}^{+}$of $T_{t, n}$. To prove convergence of global unstable manifolds, we show that the fixed point $h_{n}^{+}$of $T_{t, n}$ converges to the fixed point $h^{+}$of $T_{t}$.

Lemma 5.1. Suppose that Assumption 2.2 is satisfied. For every $v \in X^{-}$, there exists $v_{n} \in X_{n}^{-}$such that $v_{n} \rightarrow v$ in $L^{2}(D)$.

Proof. Let $v \in X^{-} \subset L^{2}(\Omega)$. By the density of $H_{0}^{1}(\Omega)$ in $L^{2}(\Omega)$ and Mosco convergence assumption, it follows from a standard diagonal procedure that there exists $\xi_{n} \in H_{0}^{1}\left(\Omega_{n}\right)$ such that $\xi_{n} \rightarrow v$ in $L^{2}(D)$ as $n \rightarrow \infty$. By Lemma 4.1, we get $P_{n}^{-} \xi_{n} \rightarrow P^{-} v=v$ in $L^{2}(D)$ as $n \rightarrow \infty$. By taking $v_{n}:=P_{n}^{-} \xi_{n}$, the lemma follows. 
Let us define $h \in Y$ by

$$
h(w):=\frac{1}{C} h^{+}(w),
$$

for all $w \in X^{+}$where $C$ is a positive constant satisfying

$$
\left\|P^{+}\right\|\left\|1-P_{n}^{+}\right\| M_{1} M_{2} \leq C
$$

for all $n \in \mathbb{N}$. Note that although $\left(1-P_{n}^{+}\right)$does not converge to $\left(1-P^{+}\right)$in $\mathscr{L}\left(L^{2}(D)\right)$ under the operator norm, we use $\left\|1-P_{n}^{+}\right\| \leq 1+\left\|P_{n}^{+}\right\|$and (28) to obtain a bound $C$ above.

In the next lemma, we obtain an approximation of $h$ by functions in $Y_{n}$.

Lemma 5.2. Let $h$ be as in (32). There exists a sequence $\left\{h_{n}\right\}$ with $h_{n} \in Y_{n}$ for each $n \in \mathbb{N}$ such that

(i) $h_{n}\left(\left.P_{n}^{+} u\right|_{\Omega_{n}}\right) \rightarrow h\left(\left.P^{+} u\right|_{\Omega}\right)$ in $L^{2}(D)$ as $n \rightarrow \infty$ for all $u \in L^{2}(D)$

(ii) for each $m \in \mathbb{N}$, we have $T_{t, n}^{m}\left(h_{n}\right)\left(\left.P_{n}^{+} u\right|_{\Omega_{n}}\right) \rightarrow T_{t}^{m}(h)\left(\left.P^{+} u\right|_{\Omega}\right)$ in $L^{2}(D)$ as $n \rightarrow \infty$ for all $u \in L^{2}(D)$.

Proof. We construct $h_{n} \in Y_{n}$ as follows. Define $h_{n}: X_{n}^{+} \rightarrow X_{n}^{-}$by

$$
h_{n}(w):=\left.\frac{1}{C}\left(1-P_{n}^{+}\right)\left(h^{+}\left(\left.P^{+} w\right|_{\Omega}\right)\right)\right|_{\Omega_{n}},
$$

for $w \in X_{n}^{+}$. It is clear that $h_{n}(0)=0$. Moreover, for $w_{1}, w_{2} \in X_{n}^{+}$, it follows from the Lipschitz continuity of $h^{+}$and the choice of $C$ in (33) that

$$
\begin{aligned}
& \left\|h_{n}\left(w_{1}\right)-h_{n}\left(w_{2}\right)\right\|_{X_{n}^{-}} \\
& \quad \leq M_{1}\left\|\left.\frac{1}{C}\left(1-P_{n}^{+}\right)\left(h^{+}\left(\left.P^{+} w_{1}\right|_{\Omega}\right)\right)\right|_{\Omega_{n}}-\left.\frac{1}{C}\left(1-P_{n}^{+}\right)\left(h^{+}\left(\left.P^{+} w_{2}\right|_{\Omega}\right)\right)\right|_{\Omega_{n}}\right\|_{L^{2}\left(\Omega_{n}\right)} \\
& \quad \leq M_{1} \frac{1}{C}\left\|1-P_{n}^{+}\right\|\left\|h^{+}\left(\left.P^{+} w_{1}\right|_{\Omega}\right)-h^{+}\left(\left.P^{+} w_{2}\right|_{\Omega}\right)\right\|_{X^{-}} \\
& \quad \leq M_{1} \frac{1}{C} \nu^{-1}\left\|1-P_{n}^{+}\right\|\left\|\left.P^{+} w_{1}\right|_{\Omega}-\left.P^{+} w_{2}\right|_{\Omega}\right\|_{X^{+}} \\
& \quad \leq M_{1} \frac{1}{C} \nu^{-1} M_{2}\left\|1-P_{n}^{+}\right\|\left\|P^{+}\right\|\left\|w_{1}-w_{2}\right\|_{L^{2}\left(\Omega_{n}\right)} \\
& \quad \leq \nu^{-1}\left\|w_{1}-w_{2}\right\|_{X_{n}^{+}} .
\end{aligned}
$$

Hence, $h_{n}$ is $\nu^{-1}$-Lipschitz and thus $h_{n} \in Y_{n}$. Note that we need to be careful about the norm used in the above calculation. In particular, we take care of the equivalence of norms on $X^{-}$and $X^{+}$given in (19). This will be applied throughout the paper.

We claim that $h_{n}$ defined above satisfies the properties (i) and (ii). For (i), let $u \in L^{2}(D)$ be arbitrary. By Lemma 5.1, there exists $\left(v_{n}\right)_{n \in \mathbb{N}}$ with $v_{n} \in X_{n}^{-}$such that

$$
v_{n} \rightarrow h^{+}\left(\left.P^{+} u\right|_{\Omega}\right)
$$


in $L^{2}(D)$ as $n \rightarrow \infty$. We have from the triangle inequality that

$$
\begin{aligned}
&\left\|h_{n}\left(\left.P_{n}^{+} u\right|_{\Omega_{n}}\right)-h\left(\left.P^{+} u\right|_{\Omega}\right)\right\|_{L^{2}(D)} \\
&=\left\|\left.\frac{1}{C}\left(1-P_{n}^{+}\right)\left(h^{+}\left(\left.P^{+}\left(\left.P_{n}^{+} u\right|_{\Omega_{n}}\right)\right|_{\Omega}\right)\right)\right|_{\Omega_{n}}-\frac{1}{C} h^{+}\left(\left.P^{+} u\right|_{\Omega}\right)\right\|_{L^{2}(D)} \\
& \leq \frac{1}{C}\left\|\left.\left(1-P_{n}^{+}\right)\left(h^{+}\left(\left.P^{+}\left(\left.P_{n}^{+} u\right|_{\Omega_{n}}\right)\right|_{\Omega}\right)\right)\right|_{\Omega_{n}}-\left.\left(1-P_{n}^{+}\right)\left(h^{+}\left(\left.P^{+} u\right|_{\Omega}\right)\right)\right|_{\Omega_{n}}\right\|_{L^{2}(D)} \\
&+\frac{1}{C}\left\|\left.\left(1-P_{n}^{+}\right)\left(h^{+}\left(\left.P^{+} u\right|_{\Omega}\right)\right)\right|_{\Omega_{n}}-h^{+}\left(\left.P^{+} u\right|_{\Omega}\right)\right\|_{L^{2}(D)} .
\end{aligned}
$$

Using the equivalence of norms on $X^{-}$and $X^{+}$, we can calculate

$$
\begin{aligned}
& \frac{1}{C}\left\|\left.\left(1-P_{n}^{+}\right)\left(h^{+}\left(\left.P^{+}\left(\left.P_{n}^{+} u\right|_{\Omega_{n}}\right)\right|_{\Omega}\right)\right)\right|_{\Omega_{n}}-\left.\left(1-P_{n}^{+}\right)\left(h^{+}\left(\left.P^{+} u\right|_{\Omega}\right)\right)\right|_{\Omega_{n}}\right\|_{L^{2}(D)} \\
& \quad \leq \frac{1}{C}\left\|1-P_{n}^{+}\right\|\left\|h^{+}\left(\left.P^{+}\left(\left.P_{n}^{+} u\right|_{\Omega_{n}}\right)\right|_{\Omega}\right)-h^{+}\left(\left.P^{+} u\right|_{\Omega}\right)\right\|_{L^{2}(D)} \\
& \quad \leq \frac{1}{C}\left\|1-P_{n}^{+}\right\|\left\|h^{+}\left(\left.P^{+}\left(\left.P_{n}^{+} u\right|_{\Omega_{n}}\right)\right|_{\Omega}\right)-h^{+}\left(\left.P^{+} P^{+} u\right|_{\Omega}\right)\right\|_{X^{-}} \\
& \quad \leq \frac{1}{C} \nu^{-1}\left\|1-P_{n}^{+}\right\|\left\|\left.P^{+}\left(\left.P_{n}^{+} u\right|_{\Omega_{n}}\right)\right|_{\Omega}-\left.P^{+} P^{+} u\right|_{\Omega}\right\|_{X^{+}} \\
& \quad \leq \frac{1}{C} \nu^{-1} M_{2}\left\|1-P_{n}^{+}\right\|\left\|P^{+}\right\|\left\|\left.P_{n}^{+} u\right|_{\Omega_{n}}-\left.P^{+} u\right|_{\Omega}\right\|_{L^{2}(D)} \\
& \quad \rightarrow 0
\end{aligned}
$$

as $n \rightarrow \infty$, where we use (28) and the boundedness of $\left\|1-P_{n}^{+}\right\|$in the last step. For the second term on the right of (36), we use (35) and $\left(1-P_{n}^{+}\right) v_{n}=v_{n}$ to obtain

$$
\begin{aligned}
\frac{1}{C} \| & \left.\left(1-P_{n}^{+}\right)\left(h^{+}\left(\left.P^{+} u\right|_{\Omega}\right)\right)\right|_{\Omega_{n}}-h^{+}\left(\left.P^{+} u\right|_{\Omega}\right) \|_{L^{2}(D)} \\
\leq & \frac{1}{C}\left\|\left.\left(1-P_{n}^{+}\right)\left(h^{+}\left(\left.P^{+} u\right|_{\Omega}\right)\right)\right|_{\Omega_{n}}-v_{n}\right\|_{L^{2}(D)} \\
& +\frac{1}{C}\left\|v_{n}-h^{+}\left(\left.P^{+} u\right|_{\Omega}\right)\right\|_{L^{2}(D)} \\
\leq & \frac{1}{C}\left\|1-P_{n}^{+}\right\|\left\|h^{+}\left(\left.P^{+} u\right|_{\Omega}\right)-v_{n}\right\|_{L^{2}(D)} \\
& +\frac{1}{C}\left\|v_{n}-h^{+}\left(\left.P^{+} u\right|_{\Omega}\right)\right\|_{L^{2}(D)} \\
\rightarrow &
\end{aligned}
$$

as $n \rightarrow \infty$. It follows from (36) - (38) that

$$
\left\|h_{n}\left(\left.P_{n}^{+} u\right|_{\Omega_{n}}\right)-h\left(\left.P^{+} u\right|_{\Omega}\right)\right\|_{L^{2}(D)} \rightarrow 0
$$

as $n \rightarrow \infty$. Since the above argument is valid for any $u \in L^{2}(D)$, statement (i) follows.

We next prove (ii) by induction on $m \in \mathbb{N}$. By part (i) of this proof, the property (ii) is true for $m=0$. For induction step, assume that

$$
T_{t, n}^{m}\left(h_{n}\right)\left(\left.P_{n}^{+} u\right|_{\Omega_{n}}\right) \rightarrow T_{t}^{m}(h)\left(\left.P^{+} u\right|_{\Omega}\right)
$$


in $L^{2}(D)$ as $n \rightarrow \infty$ for all $u \in L^{2}(D)$ holds true for $m=0,1, \ldots, k$. We need to show that

$$
T_{t, n}^{k+1}\left(h_{n}\right)\left(\left.P_{n}^{+} u\right|_{\Omega_{n}}\right) \rightarrow T_{t}^{k+1}(h)\left(\left.P^{+} u\right|_{\Omega}\right)
$$

in $L^{2}(D)$ as $n \rightarrow \infty$ for all $u \in L^{2}(D)$. Let $u \in L^{2}(D)$ be arbitrary. We set $w:=\left.P^{+} u\right|_{\Omega} \in X^{+}$and $w_{n}:=\left.P_{n}^{+} u\right|_{\Omega_{n}} \in X_{n}^{+}$. It follows from (28) that

$$
w_{n} \rightarrow w
$$

in $L^{2}(D)$ as $n \rightarrow \infty$. Since $\operatorname{graph}\left(T_{t}^{k+1}(h)\right)=\Phi_{t}\left(\operatorname{graph}\left(T_{t}^{k}(h)\right)\right.$, there exists $w_{0} \in$ $X^{+}$such that

$$
\Phi_{t}\left(w_{0} \oplus T_{t}^{k}(h)\left(w_{0}\right)\right)=w \oplus T_{t}^{k+1}(h)(w) .
$$

For each $n \in \mathbb{N}$, we define $w_{0, n}:=\left.P_{n}^{+} w_{0}\right|_{\Omega_{n}}$. Again, by (28), we have $w_{0, n} \rightarrow w_{0}$ in $L^{2}(D)$ as $n \rightarrow \infty$. Moreover, by the induction hypothesis,

$$
T_{t, n}^{k}\left(h_{n}\right)\left(w_{0, n}\right)=T_{t, n}^{k}\left(h_{n}\right)\left(\left.P_{n}^{+} w_{0}\right|_{\Omega_{n}}\right) \rightarrow T_{t}^{k}(h)\left(P^{+} w_{0}\right)=T_{t}^{k}(h)\left(w_{0}\right)
$$

in $L^{2}(D)$ as $n \rightarrow \infty$. Hence, it follows from (25) that

$$
\Phi_{t, n}\left(w_{0, n} \oplus T_{t, n}^{k}\left(h_{n}\right)\left(w_{0, n}\right)\right) \rightarrow \Phi_{t}\left(w_{0} \oplus T_{t}^{k}(h)\left(w_{0}\right)\right)=\left(w \oplus T_{t}^{k+1}(h)(w)\right)
$$

in $L^{2}(D)$ as $n \rightarrow \infty$. Since $\operatorname{graph}\left(T_{t, n}^{k+1}\left(h_{n}\right)\right)=\Phi_{t, n}\left(\operatorname{graph}\left(T_{t, n}^{k}\left(h_{n}\right)\right)\right.$, there exists $\xi_{n} \in X_{n}^{+}$such that

$$
\Phi_{t, n}\left(w_{0, n} \oplus T_{t, n}^{k}\left(h_{n}\right)\left(w_{0, n}\right)\right)=\xi_{n} \oplus T_{t, n}^{k+1}\left(h_{n}\right)\left(\xi_{n}\right),
$$

for each $n \in \mathbb{N}$. Hence,

$$
\xi_{n} \oplus T_{t, n}^{k+1}\left(h_{n}\right)\left(\xi_{n}\right) \rightarrow w \oplus T_{t}^{k+1}(h)(w)
$$

in $L^{2}(D)$ as $n \rightarrow \infty$. By Lemma 4.1, it follows from (41) that

$$
\xi_{n} \rightarrow w
$$

and

$$
T_{t, n}^{k+1}\left(h_{n}\right)\left(\xi_{n}\right) \rightarrow T_{t}^{k+1}(h)(w)
$$

in $L^{2}(D)$ as $n \rightarrow \infty$. We obtain from (40) and (42) that $\left\|\xi_{n}-w_{n}\right\|_{L^{2}(D)} \rightarrow 0$ as $n \rightarrow \infty$. Since $T_{t, n}^{k+1}\left(h_{n}\right)$ is $\nu^{-1}$-Lipschitz, it follows that

$$
\begin{aligned}
\left\|T_{t, n}^{k+1}\left(h_{n}\right)\left(\xi_{n}\right)-T_{t, n}^{k+1}\left(h_{n}\right)\left(w_{n}\right)\right\|_{L^{2}\left(\Omega_{n}\right)} & \leq\left\|T_{t, n}^{k+1}\left(h_{n}\right)\left(\xi_{n}\right)-T_{t, n}^{k+1}\left(h_{n}\right)\left(w_{n}\right)\right\|_{X_{n}^{-}} \\
& \leq \nu^{-1}\left\|\xi_{n}-w_{n}\right\|_{X_{n}^{+}} \\
& \leq \nu^{-1} M_{2}\left\|\xi_{n}-w_{n}\right\|_{L^{2}\left(\Omega_{n}\right)} \\
& \rightarrow 0
\end{aligned}
$$


as $n \rightarrow \infty$. By definitions of $w_{n}$ and $w$ together with (43) and (44), we conclude that

$$
\begin{aligned}
& \left\|T_{t, n}^{k+1}\left(h_{n}\right)\left(\left.P_{n}^{+} u\right|_{\Omega_{n}}\right)-T_{t}^{k+1}(h)\left(\left.P^{+} u\right|_{\Omega}\right)\right\|_{L^{2}(D)} \\
& =\left\|T_{t, n}^{k+1}\left(h_{n}\right)\left(w_{n}\right)-T_{t}^{k+1}(h)(w)\right\|_{L^{2}(D)} \\
& \leq\left\|T_{t, n}^{k+1}\left(h_{n}\right)\left(w_{n}\right)-T_{t, n}^{k+1}\left(h_{n}\right)\left(\xi_{n}\right)\right\|_{L^{2}(D)} \\
& \quad+\left\|T_{t, n}^{k+1}\left(h_{n}\right)\left(\xi_{n}\right)-T_{t}^{k+1}(h)(w)\right\|_{L^{2}(D)} \\
& \quad \rightarrow 0
\end{aligned}
$$

as $n \rightarrow \infty$. As $u \in L^{2}(D)$ was arbitrary, we have shown (39).

We prove the pointwise convergence of global unstable invariant manifolds in the following theorem.

Theorem 5.3. Assume that all assumptions in Theorem 2.5 are satisfied and $H_{0}^{1}\left(\Omega_{n}\right)$ converges to $H_{0}^{1}(\Omega)$ in the sense of Mosco. Then we have

$$
h_{n}^{+}\left(\left.P_{n}^{+} u\right|_{\Omega_{n}}\right) \rightarrow h^{+}\left(\left.P^{+} u\right|_{\Omega}\right)
$$

in $L^{2}(D)$ as $n \rightarrow \infty$ for all $u \in L^{2}(D)$.

Proof. Fix $u \in L^{2}(D)$ and let $\zeta>0$ be arbitrary. We can choose $m_{0} \in \mathbb{N}$ independent of $n$ such that the contraction constant $K$ in (31) satisfies

$$
\max \left\{\sup _{n \in \mathbb{N}}\left\{\frac{K^{m_{0}}}{1-K} 2 \nu^{-1}\left\|\left.P_{n}^{+} u\right|_{\Omega_{n}}\right\|_{X_{n}^{+}}\right\}, \frac{K^{m_{0}}}{1-K} 2 \nu^{-1}\left\|\left.P^{+} u\right|_{\Omega}\right\|_{X^{+}}\right\} \leq \frac{\zeta}{3} .
$$

We take $h_{n} \in Y_{n}$ and $h \in Y$ as in Lemma 5.2. Then by the definition of Lip-norm on $Y$ and $Y_{n}$ (see (24) and (30), respectively), we see that

$$
\begin{aligned}
\| h_{n}^{+} & \left(\left.P_{n}^{+} u\right|_{\Omega_{n}}\right)-h^{+}\left(\left.P^{+} u\right|_{\Omega}\right) \|_{L^{2}(D)} \\
\leq & \left\|h_{n}^{+}\left(\left.P_{n}^{+} u\right|_{\Omega_{n}}\right)-T_{t, n}^{m_{0}}\left(h_{n}\right)\left(\left.P_{n}^{+} u\right|_{\Omega_{n}}\right)\right\|_{X_{n}^{-}} \\
& +\left\|T_{t, n}^{m_{0}}\left(h_{n}\right)\left(\left.P_{n}^{+} u\right|_{\Omega_{n}}\right)-T_{t}^{m_{0}}(h)\left(\left.P^{+} u\right|_{\Omega}\right)\right\|_{L^{2}(D)} \\
& +\left\|T_{t}^{m_{0}}(h)\left(\left.P^{+} u\right|_{\Omega}\right)-h^{+}\left(\left.P^{+} u\right|_{\Omega}\right)\right\|_{X^{-}} \\
\leq & \left\|h_{n}^{+}-T_{t, n}^{m_{0}}\left(h_{n}\right)\right\|_{\text {Lip }}\left\|\left.P_{n}^{+} u\right|_{\Omega_{n}}\right\|_{X_{n}^{+}} \\
& +\left\|T_{t, n}^{m_{0}}\left(h_{n}\right)\left(\left.P_{n}^{+} u\right|_{\Omega_{n}}\right)-T_{t}^{m_{0}}(h)\left(\left.P^{+} u\right|_{\Omega}\right)\right\|_{L^{2}(D)} \\
& +\left\|T_{t}^{m_{0}}(h)-h^{+}\right\|_{\text {Lip }}\left\|\left.P^{+} u\right|_{\Omega}\right\|_{X^{+}},
\end{aligned}
$$

for all $n \in \mathbb{N}$. By an elementary result on the rate of convergence to the fixed point of a contraction mapping (see e.g. [16, Remark 1.2.3 (ii)]), we have

$$
\left\|h^{+}-T_{t}^{m_{0}}(h)\right\|_{\text {Lip }} \leq \frac{K^{m_{0}}}{1-K}\left\|h-T_{t}(h)\right\|_{\text {Lip }} \leq \frac{K^{m_{0}}}{1-K} 2 \nu^{-1}
$$

and

$$
\left\|h_{n}^{+}-T_{t, n}^{m_{0}}\left(h_{n}\right)\right\|_{\text {Lip }} \leq \frac{K^{m_{0}}}{1-K}\left\|h_{n}-T_{t, n}\left(h_{n}\right)\right\|_{\text {Lip }} \leq \frac{K^{m_{0}}}{1-K} 2 \nu^{-1},
$$

for all $n \in \mathbb{N}$. Moreover, Lemma 5.2 (ii) implies that there exists $N_{0} \in \mathbb{N}$ such that

$$
\left\|T_{t, n}^{m_{0}}\left(h_{n}\right)\left(\left.P_{n}^{+} u\right|_{\Omega_{n}}\right)-T_{t}^{m_{0}}(h)\left(\left.P^{+} u\right|_{\Omega}\right)\right\|_{L^{2}(D)} \leq \frac{\zeta}{3}
$$


for all $n>N_{0}$. It follows from (46) - (49) that

$$
\begin{aligned}
\left\|h_{n}^{+}\left(\left.P_{n}^{+} u\right|_{\Omega_{n}}\right)-h^{+}\left(\left.P^{+} u\right|_{\Omega}\right)\right\|_{L^{2}(D)} \leq & \frac{K^{m_{0}}}{1-K} 2 \nu^{-1}\left\|\left.P_{n}^{+} u\right|_{\Omega_{n}}\right\|_{X_{n}^{+}}+\frac{\zeta}{3} \\
& +\frac{K^{m_{0}}}{1-K} 2 \nu^{-1}\left\|\left.P^{+} u\right|_{\Omega}\right\|_{X^{+}},
\end{aligned}
$$

for all $n>N_{0}$. By our choice of $m_{0}$ in (45), we conclude that

$$
\left\|h_{n}^{+}\left(\left.P_{n}^{+} u\right|_{\Omega_{n}}\right)-h^{+}\left(\left.P^{+} u\right|_{\Omega}\right)\right\|_{L^{2}(D)} \leq \zeta,
$$

for all $n>N_{0}$. As $\zeta>0$ was arbitrary, we get $h_{n}^{+}\left(\left.P_{n}^{+} u\right|_{\Omega_{n}}\right) \rightarrow h^{+}\left(\left.P^{+} u\right|_{\Omega}\right)$ in $L^{2}(D)$ as $n \rightarrow \infty$. Since this argument works for any $u \in L^{2}(D)$, the statement of the theorem follows.

5.2. Upper and lower semicontinuity of local unstable manifolds. We are now in the position to prove Theorem 2.5.

Proof of Theorem 2.5 (ii). As discussed at the end of Section 3.2, there exist $\delta_{1}$ and $\delta_{2}$ such that $W_{n}^{u}=W_{n}^{u}\left(U_{n}\right)$ is a local unstable invariant manifold where $U_{n}=$ $V_{1, n} \times V_{2, n}$ with $V_{1, n}$ is a ball of radius $\delta_{1}$ in $X_{n}^{-}$and $V_{2, n}$ is a ball of radius $\delta_{2}$ in $X_{n}^{+}$ for all $n \in \mathbb{N}$. Moreover, a similar statement holds for the unperturbed problem. By the equivalence of norms on $X_{n}^{-}$and $X_{n}^{+}$with uniform parameters $\alpha$ and $\beta$, we can chose $\delta>0$ such that $B_{n}:=B_{L^{2}\left(\Omega_{n}\right)}(0, \delta) \subset V_{1, n} \times V_{2, n}$ for all $n \in \mathbb{N}$ and $B:=B_{L^{2}(\Omega)}(0, \delta) \subset V_{1} \times V_{2}$.

To prove the lower semicontinuity, we show that for every $\zeta>0$, there exists $N_{0} \in \mathbb{N}$ independent of $u \in \operatorname{graph}\left(h^{+}\right) \cap B$ such that

$$
\inf _{v \in \operatorname{graph}\left(h_{n}^{+}\right) \cap B_{n}}\|u-v\|_{L^{2}(D)}<\zeta,
$$

for all $n>N_{0}$ and for all $u \in \operatorname{graph}\left(h^{+}\right) \cap B$. Let $\zeta>0$ be arbitrary. By the Lipschitz continuity of $h^{+}: X^{+} \rightarrow X^{-}$(taking (19) into account), we have that for every $w_{0} \in X^{+}$, there exists $\rho>0$ such that

$$
\left\|\left(w \oplus h^{+}(w)\right)-\left(w_{0} \oplus h^{+}\left(w_{0}\right)\right)\right\|_{L^{2}(\Omega)}<\frac{\zeta}{2},
$$

for all $w \in B_{X^{+}}\left(w_{0}, \rho\right):=\left\{w \in X^{+}:\left\|w-w_{0}\right\|_{L^{2}(\Omega)}<\rho\right\}$. Note that $\rho$ is independent of $w_{0} \in X^{+}$. We set

$$
W:=P^{+}\left(\operatorname{graph}\left(h^{+}\right) \cap B\right)=\left\{w \in X^{+}: w \oplus h^{+}(w) \in B\right\} .
$$

Since $\operatorname{dim}\left(X^{+}\right)<\infty$, the set $\bar{W}$ is compact. Hence, we can choose a finite cover $\left\{B_{X^{+}}\left(w_{k}, \rho\right): w_{k} \in W, k=1, \ldots, m\right\}$ of $\bar{W}$ so that

$$
W \subset \bigcup_{k=1}^{m} B_{X^{+}}\left(w_{k}, \rho\right) .
$$

Denoted by $\Delta:=\min \left\{\delta-\left\|w_{k} \oplus h^{+}\left(w_{k}\right)\right\|_{L^{2}(\Omega)}: k=1, \ldots, m\right\}$. Setting $w_{k, n}:=$ $\left.P_{n}^{+} w_{k}\right|_{\Omega_{n}} \in X_{n}^{+}$for $n \in \mathbb{N}$ and $k=1, \ldots, m$. We have from (28) that $w_{k, n} \rightarrow w_{k}$ in $L^{2}(D)$ as $n \rightarrow \infty$ for each $k=1, \ldots, m$. Moreover, by Theorem $5.3 h_{n}^{+}\left(w_{k, n}\right) \rightarrow$ 
$h^{+}\left(w_{k}\right)$ in $L^{2}(D)$ as $n \rightarrow \infty$ for each $k=1, \ldots, m$. Hence, we can find $N_{0} \in \mathbb{N}$ such that

$$
\left\|\left(w_{k, n} \oplus h_{n}^{+}\left(w_{k, n}\right)\right)-\left(w_{k} \oplus h^{+}\left(w_{k}\right)\right)\right\|_{L^{2}(D)}<\min \left\{\frac{\zeta}{2}, \Delta\right\},
$$

for all $n>N_{0}$ and for all $k=1, \ldots, m$. Using (52), we have

$$
\begin{aligned}
\left\|w_{k, n} \oplus h_{n}^{+}\left(w_{k, n}\right)\right\|_{L^{2}\left(\Omega_{n}\right)} \leq & \left\|\left(w_{k, n} \oplus h_{n}^{+}\left(w_{k, n}\right)\right)-\left(w_{k} \oplus h^{+}\left(w_{k}\right)\right)\right\|_{L^{2}(D)} \\
& +\left\|w_{k} \oplus h_{n}^{+}\left(w_{k}\right)\right\|_{L^{2}(\Omega)} \\
< & \left\|w_{k} \oplus h_{n}^{+}\left(w_{k}\right)\right\|_{L^{2}(\Omega)}+\Delta \\
\leq & \left\|w_{k} \oplus h_{n}^{+}\left(w_{k}\right)\right\|_{L^{2}(\Omega)}+\left(\delta-\left\|w_{k} \oplus h_{n}^{+}\left(w_{k}\right)\right\|_{L^{2}(\Omega)}\right) \\
= & \delta,
\end{aligned}
$$

for all $n>N_{0}$ and for all $k=1, \ldots, m$. Hence, $w_{k, n} \oplus h_{n}^{+}\left(w_{k, n}\right) \in \operatorname{graph}\left(h_{n}^{+}\right) \cap B_{n}$ for all $n>N_{0}$ and for all $k=1, \ldots, m$. Let $u$ be in $\operatorname{graph}\left(h^{+}\right) \cap B$ and write $u=w \oplus h^{+}(w)$ for some $w \in W$. By (51), there exists $k \in\{1, \ldots, m\}$ such that $w \in B_{X^{+}}\left(w_{k}, \rho\right)$. It follows from (50) and (52) that

$$
\begin{aligned}
&\left\|\left(w_{k, n} \oplus h_{n}^{+}\left(w_{k, n}\right)\right)-\left(w \oplus h^{+}(w)\right)\right\|_{L^{2}(D)} \\
& \leq\left\|\left(w_{k, n} \oplus h_{n}^{+}\left(w_{k, n}\right)\right)-\left(w_{k} \oplus h^{+}\left(w_{k}\right)\right)\right\|_{L^{2}(D)} \\
&+\left\|\left(w_{k} \oplus h^{+}\left(w_{k}\right)\right)-\left(w \oplus h^{+}(w)\right)\right\|_{L^{2}(D)} \\
&< \frac{\zeta}{2}+\frac{\zeta}{2} \\
&= \zeta
\end{aligned}
$$

for all $n>N_{0}$. Since $w_{k, n} \oplus h_{n}^{+}\left(w_{k, n}\right) \in \operatorname{graph}\left(h_{n}^{+}\right) \cap B_{n}$ for all $n>N_{0}$, we get

$$
\inf _{v \in \operatorname{graph}\left(h_{n}^{+}\right) \cap B_{n}}\|u-v\|_{L^{2}(D)}<\zeta,
$$

for all $n>N_{0}$. The above estimate holds for every $u=w \oplus h^{+}(w) \in \operatorname{graph}\left(h^{+}\right) \cap B$ and notice that $N_{0}$ is independent of $u$. As $\zeta>0$ was arbitrary, we obtain the lower semicontinuity.

Using our characterisation in Lemma 4.8, we can show the upper semicontinuity of unstable invariant manifolds.

Proof of Theorem 2.5 (i). We consider the same neighbourhood $B_{n}$ and $B$ as in the proof above. Let $\left\{\xi_{n}\right\}_{n \in \mathbb{N}}$ be a sequence with $\xi_{n} \in \operatorname{graph}\left(h_{n}^{+}\right) \cap B_{n}$ and $\left(\xi_{n_{k}}\right)_{k \in \mathbb{N}}$ be an arbitrary subsequence. We write $\xi_{n_{k}}:=w_{n_{k}} \oplus h_{n_{k}}^{+}\left(w_{n_{k}}\right)$ for some $w_{n_{k}} \in X_{n_{k}}^{+}$. Since $\left\|\xi_{n_{k}}\right\|_{L^{2}\left(\Omega_{n_{k}}\right)}=\left\|w_{n_{k}} \oplus h_{n_{k}}^{+}\left(w_{n_{k}}\right)\right\|_{L^{2}\left(\Omega_{n_{k}}\right)}<\delta$ for all $k \in \mathbb{N}$, we can apply Corollary 4.6 to extract a subsequence of $\left\{w_{n_{k}}\right\}_{k \in \mathbb{N}}$ (indexed again by $n_{k}$ ) such that $w_{n_{k}} \rightarrow w$ in $L^{2}(D)$ with the limit $w \in X^{+}$. Hence, by the Lipschitz continuity of $h_{n}^{+}$and Theorem 5.3, we get

$$
\begin{aligned}
\left\|h_{n_{k}}^{+}\left(w_{n_{k}}\right)-h^{+}(w)\right\|_{L^{2}(D)} \leq & \left\|h_{n_{k}}^{+}\left(w_{n_{k}}\right)-h_{n_{k}}^{+}\left(\left.P_{n_{k}}^{+} w\right|_{\Omega_{n_{k}}}\right)\right\|_{L^{2}(D)} \\
& +\left\|h_{n_{k}}^{+}\left(\left.P_{n_{k}}^{+} w\right|_{\Omega_{n_{k}}}\right)-h^{+}(w)\right\|_{L^{2}(D)} \\
& \rightarrow 0
\end{aligned}
$$


as $k \rightarrow \infty$. If we set $u:=w \oplus h^{+}(w) \in \operatorname{graph}\left(h^{+}\right)$, then $\xi_{n_{k}} \rightarrow u$ in $L^{2}(D)$ as $k \rightarrow \infty$. Since $\left\|\xi_{n_{k}}\right\|_{L^{2}(D)}<\delta$ for all $k \in \mathbb{N}$, we get $\|u\|_{L^{2}(D)} \leq \delta$. Hence, $u \in \operatorname{graph}\left(h^{+}\right) \cap \bar{B}=\overline{\operatorname{graph}\left(h^{+}\right) \cap B}$. We can find $u_{n_{k}} \in \operatorname{graph}\left(h^{+}\right) \cap B$ such that $u_{n_{k}} \rightarrow u$ in $L^{2}(\Omega)$ as $k \rightarrow \infty$. Therefore,

$$
\left\|\xi_{n_{k}}-u_{n_{k}}\right\|_{L^{2}(D)} \leq\left\|\xi_{n_{k}}-u\right\|_{L^{2}(D)}+\left\|u-u_{n_{k}}\right\|_{L^{2}(D)} \rightarrow 0
$$

as $k \rightarrow \infty$. By Lemma 4.8, the statement in Theorem 2.5 (i) follows.

\section{Convergence of Stable invariant manifolds}

Recall that the local stable manifold is a graph of Lipschitz function $h^{-}: X^{-} \rightarrow$ $X^{+}$inside a suitable product neightbourhood of $0 \in L^{2}(\Omega)$ determined by the modification in the construction (Theorem 3.5). In this section, we prove the upper and lower semicontinuity of local stable invariant manifolds with the following modification.

Fix the renorming of $X_{n}^{-}, X_{n}^{+}, X^{-}$and $X^{+}$(see (18)) using the same parameters $\alpha$ and $\beta$ for all $n \in \mathbb{N}$. By shrinking the neighbourhood (choosing a smaller Lipschitz constant $\varepsilon$ for the nonlinear terms $f_{n}$ and $f$ ), we can make the following assumption.

Assumption 6.1. We assume that

$$
0<\mu_{0}<\inf \left\{\frac{1}{2\left(\left\|P^{+}\right\|+\left\|P^{-}\right\|\right)}, \frac{1}{2\left(\left\|P_{n}^{+}\right\|+\left\|P_{n}^{-}\right\|\right)}: n \in \mathbb{N}\right\}
$$

and

$$
\mu:=\frac{\mu_{0}}{M_{1} M_{2}}
$$

are parameters such that both $\mu_{0}$ and $\mu$ satisfy the conditions for $\mu$ in (22) and (23).

We denote the Lipschitz functions for the modification $\mu_{0}$ by $\hat{h}^{-}$and for the modification $\mu$ by $h^{-}$. Let $U$ be a smaller product neighbourhood of 0 in $L^{2}(\Omega)$ such that both modifications agree. Hence, the local stable manifold is $W^{s}(U):=$ $\operatorname{graph}\left(h^{-}\right) \cap U=\operatorname{graph}\left(\hat{h}^{-}\right) \cap U$. Similarly, for each $n \in \mathbb{N}$, we denote the Lipschitz functions for the modification $\mu_{0}$ by $\hat{h}_{n}^{-}$and for the modification $\mu$ by $h_{n}^{-}$. As discussed at the end of Section 3.2, we can take a uniform product neighbourhood $U_{n}$ of 0 in $L^{2}\left(\Omega_{n}\right)$ such that both modifications agree. Hence, the local stable manifold is $W_{n}^{s}\left(U_{n}\right):=\operatorname{graph}\left(h_{n}^{-}\right) \cap U_{n}=\operatorname{graph}\left(\hat{h}_{n}^{-}\right) \cap U_{n}$. We choose $\delta>0$ so that $\bar{B} \subset U$ and $\bar{B}_{n} \subset U_{n}$, where $B:=B_{L^{2}(\Omega)}(0, \delta)$ and $B_{n}:=B_{L^{2}\left(\Omega_{n}\right)}(0, \delta)$. Hence, $h^{-}(v)=\hat{h}^{-}(v)$ on $\bar{B}$ and $h_{n}^{-}(v)=\hat{h}_{n}^{-}(v)$ on $\bar{B}_{n}$. We prove Theorem 2.6 by taking the balls of radius $\delta$ chosen above.

Lemma 6.2. Let $\delta>0$ and $\zeta_{n}>0$ be a sequence with $\zeta_{n} \rightarrow 0$ as $n \rightarrow \infty$. We write $B:=B_{L^{2}(\Omega)}(0, \delta)$ and $B_{n}:=B_{L^{2}\left(\Omega_{n}\right)}(0, \delta)$.

(i) If $z_{n}=y_{n} \oplus h^{-}\left(y_{n}\right)$ is a sequence in $\operatorname{graph}\left(h^{-}\right)$with $z_{n} \in B_{L^{2}(\Omega)}\left(0, \delta+\zeta_{n}\right)$ for each $n \in \mathbb{N}$, then there exist a subsequence $z_{n_{k}}$ and a sequence $u_{n_{k}}$ in $\operatorname{graph}\left(h^{-}\right) \cap B$ such that $\left\|z_{n_{k}}-u_{n_{k}}\right\|_{L^{2}(\Omega)} \rightarrow 0$ as $k \rightarrow \infty$. 
(ii) If $z_{n}=y_{n} \oplus h^{-}\left(y_{n}\right)$ is a sequence with $z_{n} \in \operatorname{graph}\left(h_{n}^{-}\right) \cap B_{L^{2}\left(\Omega_{n}\right)}\left(0, \delta+\zeta_{n}\right)$ for each $n \in \mathbb{N}$, then there exist a subsequence $z_{n_{k}}$ and a sequence $u_{n_{k}}$ with $u_{n_{k}} \in \operatorname{graph}\left(h_{n_{k}}^{-}\right) \cap B_{n_{k}}$ for each $k \in \mathbb{N}$ such that $\left\|z_{n_{k}}-u_{n_{k}}\right\|_{L^{2}\left(\Omega_{n_{k}}\right)} \rightarrow 0$ as $k \rightarrow \infty$.

Proof. For assertion (i), using (53) we can fix $b>0$ such that

$$
b>\frac{1}{\left(\left\|P^{+}\right\|+\left\|P^{-}\right\|\right)^{-1}-2 \mu_{0}} .
$$

Since $\zeta_{n} \rightarrow 0$, we can find $N_{0} \in \mathbb{N}$ such that $\zeta_{n}<\delta / b$ for all $n>N_{0}$. We extract a subsequence $\zeta_{n_{k}}$ so that $\zeta_{n_{k}}<\delta / b$ for all $k \in \mathbb{N}$. Define

$$
a_{n_{k}}:=1-\frac{b \zeta_{n_{k}}}{\left\|y_{n_{k}}\right\|_{L^{2}(\Omega)}+\left\|h^{-}\left(y_{n_{k}}\right)\right\|_{L^{2}(\Omega)}},
$$

for each $k \in \mathbb{N}$. By our assumptions, $\left\|z_{n_{k}}\right\|_{L^{2}(\Omega)}=\left\|y_{n_{k}} \oplus h^{-}\left(y_{n_{k}}\right)\right\|_{L^{2}(\Omega)}<\delta+\zeta_{n_{k}}$ for all $k \in \mathbb{N}$. If $\left\|z_{n_{k}}\right\|_{L^{2}(\Omega)} \geq \delta$, then

$$
\left\|y_{n_{k}}\right\|_{L^{2}(\Omega)}+\left\|h^{-}\left(y_{n_{k}}\right)\right\|_{L^{2}(\Omega)} \geq\left\|y_{n_{k}} \oplus h^{-}\left(y_{n_{k}}\right)\right\|_{L^{2}(\Omega)} \geq \delta .
$$

Since $\zeta_{n_{k}}<\delta / b$, if $\left\|z_{n_{k}}\right\|_{L^{2}(\Omega)} \geq \delta$ we have that

$$
\frac{b \zeta_{n_{k}}}{\left\|y_{n_{k}}\right\|_{L^{2}(\Omega)}+\left\|h^{-}\left(y_{n_{k}}\right)\right\|_{L^{2}(\Omega)}}<\frac{b(\delta / b)}{\delta}=1 .
$$

It follows from (56) that $0<a_{n_{k}} \leq 1$ if $\left\|z_{n_{k}}\right\|_{L^{2}(\Omega)} \geq \delta$. For each $k \in \mathbb{N}$, we define $u_{n_{k}} \in \operatorname{graph}\left(h^{-}\right)$by

$$
u_{n_{k}}:= \begin{cases}z_{n_{k}} & \text { if }\left\|z_{n_{k}}\right\|_{L^{2}(\Omega)}<\delta \\ a_{n_{k}} y_{n_{k}} \oplus h^{-}\left(a_{n_{k}} y_{n_{k}}\right) & \text { if }\left\|z_{n_{k}}\right\|_{L^{2}(\Omega)} \geq \delta .\end{cases}
$$

Clearly, $\left\|z_{n_{k}}-u_{n_{k}}\right\|_{L^{2}(\Omega)}=0$ if $\left\|z_{n_{k}}\right\|_{L^{2}(\Omega)}<\delta$. Moreover, if $\left\|z_{n_{k}}\right\|_{L^{2}(\Omega)} \geq \delta$, then

$$
\begin{aligned}
\left\|z_{n_{k}}-u_{n_{k}}\right\|_{L^{2}(\Omega)} & =\left\|\left(y_{n_{k}} \oplus h^{-}\left(y_{n_{k}}\right)\right)-\left(a_{n_{k}} y_{n_{k}} \oplus h^{-}\left(a_{n_{k}} y_{n_{k}}\right)\right)\right\|_{L^{2}(\Omega)} \\
& \leq\left\|y_{n_{k}}-a_{n_{k}} y_{n_{k}}\right\|_{L^{2}(\Omega)}+\left\|h^{-}\left(y_{n_{k}}\right)-h^{-}\left(a_{n_{k}} y_{n_{k}}\right)\right\|_{X^{+}} \\
& \leq\left\|y_{n_{k}}-a_{n_{k}} y_{n_{k}}\right\|_{L^{2}(\Omega)}+\mu\left\|y_{n_{k}}-a_{n_{k}} y_{n_{k}}\right\|_{X^{-}} \\
& \leq\left(1+\mu M_{1}\right)\left|1-a_{n_{k}}\right|\left\|y_{n_{k}}\right\|_{L^{2}(\Omega)} \\
& \leq\left(1+\mu M_{1}\right) \frac{b \zeta_{n_{k}}}{\left\|y_{n_{k}}\right\|_{L^{2}(\Omega)}+\left\|h^{-}\left(y_{n_{k}}\right)\right\|_{L^{2}(\Omega)}} \|_{L^{2}(\Omega)} \\
& \leq\left(1+\mu M_{1}\right) b \zeta_{n_{k}} .
\end{aligned}
$$

Hence, $\left\|z_{n_{k}}-u_{n_{k}}\right\|_{L^{2}(\Omega)} \leq\left(1+\mu M_{1}\right) b \zeta_{n_{k}}$ for all $k \in \mathbb{N}$. As $\zeta_{n_{k}} \rightarrow 0$, we conclude that

$$
\left\|z_{n_{k}}-u_{n_{k}}\right\|_{L^{2}(\Omega)} \rightarrow 0
$$


as $k \rightarrow \infty$. It remains to show that $u_{n_{k}} \in B_{L^{2}(\Omega)}(0, \delta)$ for all $k \in \mathbb{N}$. If $\left\|z_{n_{k}}\right\|_{L^{2}(\Omega)}<$ $\delta$, then $u_{n_{k}} \in B_{L^{2}(\Omega)}(0, \delta)$. If $\left\|z_{n_{k}}\right\|_{L^{2}(\Omega)} \geq \delta$, we can write

$$
\begin{aligned}
\left\|u_{n_{k}}\right\|_{L^{2}(\Omega)} \leq & \left\|u_{n_{k}}-a_{n_{k}} z_{n_{k}}\right\|_{L^{2}(\Omega)}+\left\|a_{n_{k}} z_{n_{k}}\right\|_{L^{2}(\Omega)} \\
= & \left\|\left(a_{n_{k}} y_{n_{k}} \oplus h^{-}\left(a_{n_{k}} y_{n_{k}}\right)\right)-a_{n_{k}}\left(y_{n_{k}} \oplus h^{-}\left(y_{n_{k}}\right)\right)\right\|_{L^{2}(\Omega)} \\
& +\left\|a_{n_{k}} z_{n_{k}}\right\|_{L^{2}(\Omega)} \\
\leq & \left\|h^{-}\left(a_{n_{k}} y_{n_{k}}\right)-a_{n_{k}} h^{-}\left(y_{n_{k}}\right)\right\|_{L^{2}(\Omega)}+\left\|a_{n_{k}} z_{n_{k}}\right\|_{L^{2}(\Omega)} \\
\leq & \left\|h^{-}\left(a_{n_{k}} y_{n_{k}}\right)-h^{-}\left(y_{n_{k}}\right)\right\|_{L^{2}(\Omega)}+\left\|h^{-}\left(y_{n_{k}}\right)-a_{n_{k}} h^{-}\left(y_{n_{k}}\right)\right\|_{L^{2}(\Omega)} \\
& +\left\|a_{n_{k}} z_{n_{k}}\right\|_{L^{2}(\Omega)} .
\end{aligned}
$$

Now, if $\left\|z_{n_{k}}\right\|_{L^{2}(\Omega)} \geq \delta$, then by the Lipschitz continuity of $h^{-}$and (54)

$$
\begin{aligned}
\left\|h^{-}\left(a_{n_{k}} y_{n_{k}}\right)-h^{-}\left(y_{n_{k}}\right)\right\|_{L^{2}(\Omega)} & \leq\left\|h^{-}\left(a_{n_{k}} y_{n_{k}}\right)-h^{-}\left(y_{n_{k}}\right)\right\|_{X^{+}} \\
& \leq \mu\left\|a_{n_{k}} y_{n_{k}}-y_{n_{k}}\right\|_{X^{-}} \\
& \leq \mu M_{1}\left|a_{n_{k}}-1\right|\left\|y_{n_{k}}\right\|_{L^{2}(\Omega)} \\
& =\frac{\mu_{0}}{M_{1} M_{2}} M_{1} \frac{b \zeta_{n_{k}}\left\|y_{n_{k}}\right\|_{L^{2}(\Omega)}}{\left\|y_{n_{k}}\right\|_{L^{2}(\Omega)}+\left\|h^{-}\left(y_{n_{k}}\right)\right\|_{L^{2}(\Omega)}} \\
& \leq \mu_{0} b \zeta_{n_{k}} .
\end{aligned}
$$

Similarly, if $\left\|z_{n_{k}}\right\|_{L^{2}(\Omega)} \geq \delta$, then

$$
\begin{aligned}
\left\|h^{-}\left(y_{n_{k}}\right)-a_{n_{k}} h^{-}\left(y_{n_{k}}\right)\right\|_{L^{2}(\Omega)} & \leq\left|1-a_{n_{k}}\right|\left\|h^{-}\left(y_{n_{k}}\right)\right\|_{X^{+}} \\
& \leq \mu\left|1-a_{n_{k}}\right|\left\|y_{n_{k}}\right\|_{X^{-}} \\
& \leq \mu M_{1}\left|1-a_{n_{k}}\right|\left\|y_{n_{k}}\right\|_{L^{2}(\Omega)} \\
& =\frac{\mu_{0}}{M_{1} M_{2}} M_{1} \frac{b \zeta_{n_{k}}\left\|y_{n_{k}}\right\|_{L^{2}(\Omega)}}{\left\|y_{n_{k}}\right\|_{L^{2}(\Omega)}+\left\|h^{-}\left(y_{n_{k}}\right)\right\|_{L^{2}(\Omega)}} \\
& \leq \mu_{0} b \zeta_{n_{k}} .
\end{aligned}
$$

Since $\left\|z_{n_{k}}\right\|_{L^{2}(\Omega)} \geq\left(\left\|y_{n_{k}}\right\|_{L^{2}(\Omega)}+\left\|h^{-}\left(y_{n_{k}}\right)\right\|_{L^{2}(\Omega)}\right) /\left(\left\|P^{+}\right\|+\left\|P^{-}\right\|\right)$, it follows that

$$
\frac{b \zeta_{n_{k}}\left\|z_{n_{k}}\right\|_{L^{2}(\Omega)}}{\left\|y_{n_{k}}\right\|_{L^{2}(\Omega)}+\left\|h^{-}\left(y_{n_{k}}\right)\right\|_{L^{2}(\Omega)}} \geq \frac{b \zeta_{n_{k}}}{\left\|P^{+}\right\|+\left\|P^{-}\right\|} .
$$

Hence, if $\left\|z_{n_{k}}\right\|_{L^{2}(\Omega)} \geq \delta$, then

$$
\begin{aligned}
\left\|a_{n_{k}} z_{n_{k}}\right\|_{L^{2}(\Omega)} & =\left(1-\frac{b \zeta_{n_{k}}}{\left\|y_{n_{k}}\right\|_{L^{2}(\Omega)}+\left\|h^{-}\left(y_{n_{k}}\right)\right\|_{L^{2}(\Omega)}}\right)\left\|z_{n_{k}}\right\|_{L^{2}(\Omega)} \\
& =\left\|z_{n_{k}}\right\|_{L^{2}(\Omega)}-\frac{b \zeta_{n_{k}}\left\|z_{n_{k}}\right\|_{L^{2}(\Omega)}}{\left\|y_{n_{k}}\right\|_{L^{2}(\Omega)}+\left\|h^{-}\left(y_{n_{k}}\right)\right\|_{L^{2}(\Omega)}} \\
& \leq\left\|z_{n_{k}}\right\|_{L^{2}(\Omega)}-\frac{b \zeta_{n_{k}}}{\left\|P^{+}\right\|+\left\|P^{-}\right\|} \\
& <\delta+\zeta_{n_{k}}-\frac{b \zeta_{n_{k}}}{\left\|P^{+}\right\|+\left\|P^{-}\right\|}
\end{aligned}
$$


Therefore, by (59) - (62), if $\left\|z_{n_{k}}\right\|_{L^{2}(\Omega)} \geq \delta$, then

$$
\begin{aligned}
\left\|u_{n_{k}}\right\|_{L^{2}(\Omega)} & <\mu_{0} b \zeta_{n_{k}}+\mu_{0} b \zeta_{n_{k}}+\delta+\zeta_{n_{k}}-\frac{b \zeta_{n_{k}}}{\left\|P^{+}\right\|+\left\|P^{-}\right\|} \\
& =\delta+\left(2 \mu_{0} b-\frac{b}{\left\|P^{+}\right\|+\left\|P^{-}\right\|}+1\right) \zeta_{n_{k}} .
\end{aligned}
$$

By the choice of $b$ in (55), we get

$$
\begin{aligned}
2 \mu_{0} b-\frac{b}{\left\|P^{+}\right\|+\left\|P^{-}\right\|}+1 & =-\left(\left(\left\|P^{+}\right\|+\left\|P^{-}\right\|\right)^{-1}-2 \mu_{0}\right) b+1 \\
& <-1+1 \\
& =0 .
\end{aligned}
$$

It follows from (63) that $\left\|u_{n_{k}}\right\|_{L^{2}(\Omega)}<\delta$ if $\left\|z_{n_{k}}\right\|_{L^{2}(\Omega)} \geq \delta$. Hence, we conclude that $u_{n_{k}} \in \operatorname{graph}\left(h^{-}\right) \cap B_{L^{2}(\Omega)}(0, \delta)$ for all $k \in \mathbb{N}$ and statement (i) follows.

Statement (ii) can be proved similarly. The only difference is that the sequence $z_{n}$ belongs to different spaces $L^{2}\left(\Omega_{n}\right)$ for each $n \in \mathbb{N}$. We only need to adjust the proof in part (i) and keep track of the dependence on $n$. In particular, we replace (55) by

and (56) by

$$
b>\sup _{n \in \mathbb{N}}\left\{\frac{1}{\left(\left\|P_{n}^{+}\right\|+\left\|P_{n}^{-}\right\|\right)^{-1}-2 \mu_{0}}\right\}>0
$$

$$
a_{n_{k}}:=1-\frac{b \zeta_{n_{k}}}{\left\|y_{n_{k}}\right\|_{L^{2}\left(\Omega_{n_{k}}\right)}+\left\|h_{n_{k}}^{-}\left(y_{n_{k}}\right)\right\|_{L^{2}\left(\Omega_{n_{k}}\right)}},
$$

for each $k \in \mathbb{N}$.

We now show the upper semicontinuity of local stable invariant manifolds.

Proof of Theorem 2.6 (i). By Lemma 4.8, we need to show that for any sequence $\left\{\xi_{n}\right\}_{n \in \mathbb{N}}$ with $\xi_{n} \in \operatorname{graph}\left(h_{n}^{-}\right) \cap B_{n}$, if $\left\{\xi_{n_{k}}\right\}_{k \in \mathbb{N}}$ is a subsequence then there exist a further subsequence (denoted again by $\xi_{n_{k}}$ ) and a sequence $\left\{u_{n_{k}}\right\}_{k \in \mathbb{N}}$ with $u_{n_{k}} \in$ $\operatorname{graph}\left(h^{-}\right) \cap B$ such that $\left\|\xi_{n_{k}}-u_{n_{k}}\right\|_{L^{2}(D)} \rightarrow 0$ as $k \rightarrow \infty$.

Let $\left\{\xi_{n}\right\}_{n \in \mathbb{N}}$ be a sequence with $\xi_{n} \in \operatorname{graph}\left(h_{n}^{-}\right) \cap B_{n}$ and $\left(\xi_{n_{k}}\right)_{k \in \mathbb{N}}$ be an arbitrary subsequence. We write $\xi_{n_{k}}:=v_{n_{k}} \oplus h_{n_{k}}^{-}\left(v_{n_{k}}\right)$ for some $v_{n_{k}} \in X_{n_{k}}^{-}$. Since $\left\|\xi_{n_{k}}\right\|_{L^{2}\left(\Omega_{n_{k}}\right)}=\left\|v_{n_{k}} \oplus h_{n_{k}}^{-}\left(v_{n_{k}}\right)\right\|_{L^{2}\left(\Omega_{n_{k}}\right)}<\delta$ for all $k \in \mathbb{N}$, we can extract a subsequence of $v_{n_{k}}$ (indexed again by $n_{k}$ ) such that

$$
v_{n_{k}} \rightarrow v
$$

in $L^{2}(D)$ as $k \rightarrow \infty$. By the assumption that $\left|\Omega_{n}\right| \rightarrow|\Omega|$, we conclude that $v=0$ almost everywhere in $D \backslash \Omega$, that is, $v \in L^{2}(\Omega)$. Moreover, by the convergence of $P_{n}^{-} \rightarrow P^{-}$in $\mathscr{L}\left(L^{2}(D)\right)$ (see Remark 4.2) and the weak convergence of $v_{n_{k}}$, it is easy to see that $v_{n_{k}} \rightarrow P^{-} v$ in $L^{2}(D)$ as $k \rightarrow \infty$. By the uniqueness of weak limit, $v=P^{-} v$ and hence $v \in X^{-}$. Since $\left\|h_{n_{k}}^{-}\left(v_{n_{k}}\right)\right\|_{L^{2}(D)}$ is uniformly bounded, we can apply Corollary 4.6 to extract a further subsequence (indexed again by $n_{k}$ ) such that

$$
h_{n_{k}}^{-}\left(v_{n_{k}}\right) \rightarrow w
$$


in $L^{2}(D)$ as $k \rightarrow \infty$ with the limit $w \in X^{+}$. Thus, we get

$$
v_{n_{k}} \oplus h_{n_{k}}^{-}\left(v_{n_{k}}\right) \rightarrow v \oplus w
$$

in $L^{2}(D)$ as $k \rightarrow \infty$. By a standard property of weak convergence,

$$
\|v \oplus w\|_{L^{2}(D)} \leq \liminf _{k \rightarrow \infty}\left\|v_{n_{k}} \oplus h_{n_{k}}^{-}\left(v_{n_{k}}\right)\right\|_{L^{2}(D)} \leq \delta .
$$

Hence, $u:=v \oplus w$ belongs to $\bar{B}$. Applying (25), we get from (66) and globally Lipschitz assumption for the modified function $\tilde{f}$ that $\Phi_{t, n_{k}}\left(v_{n_{k}} \oplus h_{n_{k}}^{-}\left(v_{n_{k}}\right)\right) \rightarrow$ $\Phi_{t}(v \oplus w)$ in $L^{2}(D)$ as $k \rightarrow \infty$ for all $t>0$. Lemma 4.1 implies that

$$
\begin{aligned}
P_{n_{k}}^{-} \Phi_{t, n_{k}}\left(v_{n_{k}} \oplus h_{n_{k}}^{-}\left(v_{n_{k}}\right)\right), & \rightarrow P^{-} \Phi_{t}(v \oplus w) \\
P_{n_{k}}^{+} \Phi_{t, n_{k}}\left(v_{n_{k}} \oplus h_{n_{k}}^{-}\left(v_{n_{k}}\right)\right) & \rightarrow P^{+} \Phi_{t}(v \oplus w)
\end{aligned}
$$

in $L^{2}(D)$ as $k \rightarrow \infty$ for all $t>0$. By the construction of $h_{n_{k}}^{-}\left(v_{n_{k}}\right)$ (see Theorem $3.2)$, we have that

$$
\left\|P_{n_{k}}^{+} \Phi_{t, n_{k}}\left(v_{n_{k}} \oplus h_{n_{k}}^{-}\left(v_{n_{k}}\right)\right)\right\|_{X_{n_{k}}^{+}} \leq \mu\left\|P_{n_{k}}^{-} \Phi_{t, n_{k}}\left(v_{n_{k}} \oplus h_{n_{k}}^{-}\left(v_{n_{k}}\right)\right)\right\|_{X_{n_{k}}^{-}},
$$

for all $t \geq 0$. The above implies

$$
\left\|P_{n_{k}}^{+} \Phi_{t, n_{k}}\left(v_{n_{k}} \oplus h_{n_{k}}^{-}\left(v_{n_{k}}\right)\right)\right\|_{L^{2}\left(\Omega_{n_{k}}\right)} \leq \mu M_{1}\left\|P_{n_{k}}^{-} \Phi_{t, n_{k}}\left(v_{n_{k}} \oplus h_{n_{k}}^{-}\left(v_{n_{k}}\right)\right)\right\|_{L^{2}\left(\Omega_{n_{k}}\right)},
$$

for all $t \geq 0$. Passing to the limit as $k \rightarrow \infty$, we obtain

$$
\left\|P^{+} \Phi_{t}(v \oplus w)\right\|_{L^{2}(\Omega)} \leq \mu M_{1}\left\|P^{-} \Phi_{t}(v \oplus w)\right\|_{L^{2}(\Omega)}
$$

for all $t>0$. By the assumptions on $\mu_{0}$ and $\mu$ in (53) and (54), and the equivalence of norms on $X^{-}$and $X^{+}$, it follows that

$$
\left\|P^{+} \Phi_{t}(v \oplus w)\right\|_{X^{+}} \leq \mu M_{1} M_{2}\left\|P^{-} \Phi_{t}(v \oplus w)\right\|_{X^{-}}=\mu_{0}\left\|P^{-} \Phi_{t}(v \oplus w)\right\|_{X^{-}},
$$

for all $t>0$. We claim that $\|w\|_{X^{+}} \leq \mu_{0}\|v\|_{X^{-}}$. If $\|w\|_{X^{+}}>\mu_{0}\|v\|_{X^{-}}$, that is $v \oplus w$ is in the interior of the cone $K_{\mu_{0}}$ defined by (21), we can find a product neighbourhood $U(v, w)$ of $v \oplus w \operatorname{such}$ that $U(v, w) \subset \operatorname{Int}\left(K_{\mu_{0}}\right)$. Since the solution of parabolic equation with the initial condition $v \oplus w$ is continuous, there exists $t_{0}>0$ such that $\Phi_{t}(v \oplus w) \in U(v, w)$ for $0 \leq t \leq t_{0}$. This implies that $\left\|P^{+} \Phi_{t}(v \oplus w)\right\|_{X^{+}}>$ $\mu_{0}\left\|P^{-} \Phi_{t}(v \oplus w)\right\|_{X^{-}}$for $0 \leq t \leq t_{0}$, which is a contradiction to (68). Hence, by the definition of $\hat{h}^{-}$(a modification with the cone $K_{\mu_{0}}$ ), we conclude that $w=\hat{h}^{-}(v)$. As both modification agree on $\bar{B}$, we have $w=h^{-}(v)$. Therefore, (65) implies

$$
h_{n_{k}}^{-}\left(v_{n_{k}}\right) \rightarrow h^{-}(v)
$$

in $L^{2}(D)$ as $k \rightarrow \infty$.

The remainder of this proof deals with the existence of the required sequence $u_{n_{k}} \in \operatorname{graph}\left(h^{-}\right) \cap B$. At this stage, we keep the index of our subsequence as in the previous part. We define $y_{n_{k}}:=\left.P^{-} v_{n_{k}}\right|_{\Omega} \in X^{-}$for each $k \in \mathbb{N}$. By the convergence $P_{n}^{-} \rightarrow P^{-}$in $\mathscr{L}\left(L^{2}(D)\right)$ (from Remark 4.2) and the boundedness of $\left\|v_{n_{k}}\right\|_{L^{2}(D)}$, we get

$$
\left\|y_{n_{k}}-v_{n_{k}}\right\|_{L^{2}(D)} \leq\left\|P^{-}-P_{n_{k}}^{-}\right\|\left\|v_{n_{k}}\right\|_{L^{2}(D)} \rightarrow 0
$$


as $k \rightarrow \infty$. In particular, $\left\|y_{n_{k}}\right\|_{L^{2}(\Omega)}$ is uniformly bounded. Moreover, by (70) and (64), we get

$$
y_{n_{k}} \rightarrow v
$$

in $L^{2}(D)$ as $k \rightarrow \infty$. By the Lipschitz continuity of $h^{-},\left\|h^{-}\left(y_{n_{k}}\right)\right\|_{L^{2}(\Omega)}$ is uniformly bounded. Since $X^{+}$is a finite dimensional space, we can extract a further subsequence (indexed again by $n_{k}$ ) such that

$$
h^{-}\left(y_{n_{k}}\right) \rightarrow \tilde{w}
$$

in $L^{2}(D)$ as $k \rightarrow \infty$ with the limit $\tilde{w} \in X^{+}$. Therefore, $y_{n_{k}} \oplus h^{-}\left(y_{n_{k}}\right) \rightarrow v \oplus \tilde{w}$ in $L^{2}(D)$ as $k \rightarrow \infty$. By (25) (with $\Omega_{n}=\Omega$ for all $n \in \mathbb{N}$ ), it follows that $\Phi_{t}\left(y_{n_{k}} \oplus h^{-}\left(y_{n_{k}}\right)\right) \rightarrow \Phi_{t}(v \oplus \tilde{w})$ in $L^{2}(D)$ as $k \rightarrow \infty$ for all $t>0$. Hence,

$$
\begin{aligned}
& P^{-} \Phi_{t}\left(y_{n_{k}} \oplus h^{-}\left(y_{n_{k}}\right)\right) \rightarrow P^{-} \Phi_{t}(v \oplus \tilde{w}), \\
& P^{+} \Phi_{t}\left(y_{n_{k}} \oplus h^{-}\left(y_{n_{k}}\right)\right) \rightarrow P^{+} \Phi_{t}(v \oplus \tilde{w})
\end{aligned}
$$

in $L^{2}(\Omega)$ as $k \rightarrow \infty$ for all $t>0$. Since these sequences are in the fixed spaces $X^{-}$ and $X^{+}$respectively, (19) implies that they converge under $\|\cdot\|_{X^{-}}$and $\|\cdot\|_{X^{+}}$, respectively. By the construction of $h^{-}\left(y_{n_{k}}\right)$ (see Theorem 3.2), we have that

$$
\left\|P^{+} \Phi_{t}\left(y_{n_{k}} \oplus h^{-}\left(y_{n_{k}}\right)\right)\right\|_{X^{+}} \leq \mu\left\|P^{-} \Phi_{t}\left(y_{n_{k}} \oplus h^{-}\left(y_{n_{k}}\right)\right)\right\|_{X^{-}},
$$

for all $t \geq 0$. Passing to the limit as $k \rightarrow \infty$, we obtain

$$
\left\|P^{+} \Phi_{t}(v \oplus \tilde{w})\right\|_{X^{+}}=\mu\left\|P^{-} \Phi_{t}(v \oplus \tilde{w})\right\|_{X^{-}}
$$

for all $t>0$. By a similar argument appeared after (68), we conclude that $\|\tilde{w}\|_{X^{+}} \leq$ $\mu\|v\|_{X^{-}}$. Hence, $\tilde{w}$ agrees with $w=h^{-}(v)$. Therefore, (72) implies

$$
h^{-}\left(y_{n_{k}}\right) \rightarrow h^{-}(v)
$$

in $L^{2}(D)$ as $k \rightarrow \infty$. Recall that $\xi_{n_{k}}=v_{n_{k}} \oplus h_{n_{k}}^{-}\left(v_{n_{k}}\right)$. If we set $z_{n_{k}}:=y_{n_{k}} \oplus$ $h^{-}\left(y_{n_{k}}\right) \in \operatorname{graph}\left(h^{-}\right)$, then by (69), (70) and (74), we get

$$
\begin{aligned}
\| \xi_{n_{k}} & -z_{n_{k}} \|_{L^{2}(D)} \\
= & \left\|\left(v_{n_{k}} \oplus h_{n_{k}}^{-}\left(v_{n_{k}}\right)\right)-\left(y_{n_{k}} \oplus h^{-}\left(y_{n_{k}}\right)\right)\right\|_{L^{2}(D)} \\
\leq & \left\|v_{n_{k}}-y_{n_{k}}\right\|_{L^{2}(D)}+\left\|h_{n_{k}}^{-}\left(v_{n_{k}}\right)-h^{-}\left(y_{n_{k}}\right)\right\|_{L^{2}(D)} \\
\leq & \left\|v_{n_{k}}-y_{n_{k}}\right\|_{L^{2}(D)}+\left\|h_{n_{k}}^{-}\left(v_{n_{k}}\right)-h^{-}(v)\right\|_{L^{2}(D)} \\
& \quad+\left\|h^{-}(v)-h^{-}\left(y_{n_{k}}\right)\right\|_{L^{2}(D)} \\
& \rightarrow 0
\end{aligned}
$$

as $k \rightarrow \infty$. Therefore, we can extract a further subsequence (indexed again by $n_{k}$ ) and $\zeta_{n_{k}}>0$ with $\zeta_{n_{k}} \rightarrow 0$ as $k \rightarrow \infty$ such that $\left\|\xi_{n_{k}}-z_{n_{k}}\right\|_{L^{2}(D)}<\zeta_{n_{k}}$ for all $k \in \mathbb{N}$. It follows that

$$
\left\|z_{n_{k}}\right\|_{L^{2}(\Omega)} \leq\left\|\xi_{n_{k}}\right\|_{L^{2}\left(\Omega_{n_{k}}\right)}+\zeta_{n_{k}}<\delta+\zeta_{n_{k}},
$$

for all $k \in \mathbb{N}$, that is, $z_{n_{k}} \in \operatorname{graph}\left(h^{-}\right) \cap B_{L^{2}(\Omega)}\left(0, \delta+\zeta_{n_{k}}\right)$ for all $k \in \mathbb{N}$. We can apply Lemma 6.2 (i) to obtain a subsequence (indexed again by $n_{k}$ ) $z_{n_{k}}$ and 
a sequence $u_{n_{k}} \in \operatorname{graph}\left(h^{-}\right) \cap B$ such that $\left\|z_{n_{k}}-u_{n_{k}}\right\|_{L^{2}(\Omega)} \rightarrow 0$ as $k \rightarrow \infty$. It follows from (75) that

$$
\left\|\xi_{n_{k}}-u_{n_{k}}\right\|_{L^{2}(D)} \leq\left\|\xi_{n_{k}}-z_{n_{k}}\right\|_{L^{2}(D)}+\left\|z_{n_{k}}-u_{n_{k}}\right\|_{L^{2}(D)} \rightarrow 0
$$

as $k \rightarrow \infty$. Hence, we obtain the required sequence $u_{n_{k}}$. Since we start with an arbitrary sequence $\xi_{n} \in \operatorname{graph}\left(h_{n}^{-}\right) \cap B_{n}$, the assertion of Theorem 2.6 (i) follows.

The lower semicontinuity of local stable invariant manifolds can be obtained by a similar fashion.

Proof of Theorem 2.6 (ii). The statement follows by a similar argument to the proof of Theorem 2.6 (i). We use Lemma 4.9 and Lemma 6.2 (ii) instead of Lemma 4.8 and $6.2(\mathrm{i})$.

Acknowledgment. The author would like to thank D. Daners and E. N. Dancer for helpful discussions and suggestions.

\section{REFERENCES}

[1] J. Appell and P. P. Zabrejko. Nonlinear superposition operators, volume 95 of Cambridge Tracts in Mathematics. Cambridge University Press, Cambridge, 1990.

[2] W. Arendt. Approximation of degenerate semigroups. Taiwanese J. Math., 5(2):279-295, 2001.

[3] J. M. Arrieta and A. N. Carvalho. Spectral convergence and nonlinear dynamics of reactiondiffusion equations under perturbations of the domain. J. Differential Equations, 199(1):143178, 2004.

[4] P. W. Bates and C. K. R. T. Jones. Invariant manifolds for semilinear partial differential equations. In Dynamics reported, Vol. 2, volume 2 of Dynam. Report. Ser. Dynam. Systems Appl., pages 1-38. Wiley, Chichester, 1989.

[5] D. Daners. Domain perturbation for linear and nonlinear parabolic equations. J. Differential Equations, 129(2):358-402, 1996.

[6] D. Daners. Dirichlet problems on varying domains. J. Differential Equations, 188(2):591-624, 2003.

[7] D. Daners. Perturbation of semi-linear evolution equations under weak assumptions at initial time. J. Differential Equations, 210(2):352-382, 2005.

[8] R. Dautray and J.-L. Lions. Mathematical analysis and numerical methods for science and technology. Vol. 5. Springer-Verlag, Berlin, 1992.

[9] E. A. M. de Abreu and A. N. Carvalho. Attractors for semilinear parabolic problems with Dirichlet boundary conditions in varying domains. Mat. Contemp., 27:37-51, 2004.

[10] J. Hadamard. Sur l'équilibre des plaques élastiques circulaires libres ou appuyées et celui de la sphère isotrope. Ann. Sci. École Norm. Sup. (3), 18:313-342, 1901.

[11] D. Henry. Geometric theory of semilinear parabolic equations, volume 840 of Lecture Notes in Mathematics. Springer-Verlag, Berlin, 1981.

[12] T. Kato. Perturbation theory for linear operators. Springer-Verlag, Berlin, second edition, 1976.

[13] U. Mosco. Convergence of convex sets and of solutions of variational inequalities. Advances in Math., 3:510-585, 1969.

[14] A. Pazy. Semigroups of linear operators and applications to partial differential equations, volume 44 of Applied Mathematical Sciences. Springer-Verlag, New York, 1983. 
[15] M. Prizzi and K. P. Rybakowski. The effect of domain squeezing upon the dynamics of reaction-diffusion equations. J. Differential Equations, 173(2):271-320, 2001.

[16] D. R. Smart. Fixed point theorems. Cambridge University Press, London, 1974. 